\title{
Quantitative Character Dynamics: Gametic Model
}

\author{
A. Gimelfarb \\ Department of Biostatistics, School of Public Health, \\ University of Michigan, Ann Arbor, Michigan 48109
}

Received March 1, 1982

\begin{abstract}
A gametic model of quantitative character dynamics is introduced that fills the gap between the two existing models: genic and zygotic/phenotypic. In this model, a gamete is treated as the elementary unit of evolution, all biological processes at the levels below gametic remain unspecified, and a gamete is characterized by its effect on the quantitative character rather than by the genotype. The hereditary and developmental processes are accounted for in a generalized form by gametogenetic and developmental functions defined for a pair of gametic effects representing an individual. A parameterization of these functions is suggested that imposes constraints on the heredity of quantitative characters similar to the constraints imposed by traditional genic models. It is shown that this parameterization can be derived for some polygenic additive models. General expressions for the dynamics of the mean and variance of additive quantitative characters are obtained, and the dynamics under random mating for sex-independent, sex-controlled, and sex-linked characters are considered. Comparisons with the dynamics predicted by genic models are made.
\end{abstract}

\section{INTRODUCTION}

Evolutionary models can be classified according to the elementary unit of evolution they deal with (Lewontin, 1974). By this classification, the current models of the quantitative character dynamics fall into one of three types: "genic," "zygotic," and "phenotypic."

Genic models deal with the gene as the elementary unit. Their origin goes back to the pioneer works on population genetics by Fisher, Haldane, and Wright. Models of this type may be referred to as "micro" models, since they are based on a detailed description of the evolution of the entire genetic system of the quantitative character, and the character dynamics are derived as a consequence of the evolution of the whole genetic system. The rules of heredity and development of a character are described in terms of the effects of individual genes. Since the number of genes controlling a quantitative character can be large, the number of parameters and variables needed to describe the entire genetic system of a character can be extremely large, and an obvious drawback of genic models is their often enormous combinatorial 
complexity. There are two ways of coping with this: to make simplified, sometimes oversimplified, and almost never experimentally justified assumptions about the properties of the genetic systems of quantitative characters, or to use computer simulations of the genetic systems (see, e.g., Wright, 1921a, b; Lewontin, 1964a, b; Crow and Felsenstein, 1968; Latter, 1970, 1972; Bulmer, 1971, 1972, 1973; Karlin, 1977, 1978; Karlin and Liberman, 1979). The last word in genic models is the model introduced by Lande $(1976 \mathrm{a}, \mathrm{b})$ based on a result of Kimura (1965) about the normal distribution of allelic effects within a locus with an infinite number of alleles maintained by interactions between mutations of small effect and stabilizing selection. Using this result, Lande suggested a multilocus model assuming that allelic effects are distributed normally within each of the loci. If it were the only assumption, the complexity of this model would not be reduced as compared to the "classical" diallelic, multilocus models. The crucial assumption making this model analytically and numerically manageable is that the joint distribution of allelic effects from all loci within a gamete is multivariate normal. Unlike the assumption about the normal distribution of allelic effects within one locus, this one remains unjustified, although attempts have been made to evaluate its appropriateness as an approximation (Felsenstein, 1977; Fleming, 1979).

It should be noted that the complexity of the genetic systems that quantitative characters may possess makes it virtually impossible even to imagine any practical experiments or observations that would allow one to ascertain a genic model for a real quantitative character unambiguously and to determine its parameters with reasonable accuracy. Feldman and CavalliSforza (1981) are perfectly right in their assertion that the acceptability of these models "is determined not by empirical validation (which is nigh impossible) but by the apparent preference exhibited by a few investigators." Genic models, however, have an important advantage. Given that the model's parameters are known, they may be assumed to remain unchanged during rclatively long evolutionary time, since these parameters are supposed to be determined exclusively by the biological mechanisms of heredity and development, and as such to be independent of the population structure. Therefore, genic models are useful for investigation general theoretical aspects of long-term effects on quantitiative characters of different evolutionary forces, whereas their usefulness for making predictions about actual dynamics of a real quantitative character is highly problematic.

The two other types of models of the quantitative character dynamics, currently in the literature, may be called "zygotic" or "phenotypic," depending on whether the elementary unit of evolution is the zygote or the phenotype of an individual. In zygotic models, the individual's phenotype is partitioned into components: biologically inherited, called the genotypic value (culturally inherited component also is often considered) and 
nonhereditary or environmental component. The rules of character heredity and development are described in these models in terms of the genotypic values, and all biological processes at levels below zygotic are not specified. In phenotypic models, the individual's phenotype is not partitioned, and the rules of heredity and development are described directly in terms of the individual's phenotypes with all biological processes at levels below phenotypic being unspecified. Zygotic models were introduced by CavalliSforza and Feldman (1976), and have been used by them (Cavalli-Sforza and Feldman (1976), and have been used by them (Cavalli-Sforza and Feldman, 1978; Feldman and Cavalli-Sforza, 1977, 1979) and by Rice et al. (1978; also, Cloninger et al., 1979) in the works on the "complex transmission" of quantitative characters. A phenotypic model has appeared in a work by Slatkin (1970) (also Gimelfarb, 1970), and a rigorous and extensive treatment of models of this type was given in a series of papers by Karlin (1979a-d; 1980a, b). Models of this type were applied by Rocklin and Oster (1976) to the problem of phenotype-dependent interspecific competition and by Slatkin and Lande (1976) to the problem of niche width in a fluctuating environment.

Both zygotic and phenotypic models may be regarded as "macro" models of the quantitative character dynamics, and, due to their similarity, they may be combined into one "zygotic/phenotypic" type. The following brief overview of zygotic models is almost totally related to the phenotypic ones.

It is usually assumed in these models that the genotypic values as well as the phenotypes of individuals are normally distributed in any generation. All hereditary and developmental processes, except for the environmental influences in zygotic models, are supposed to be accounted for in a generalized form by a function $L(z \mid x, y)$ describing the distribution of the genotypic values among the offspring of parents with genotypic values $x$ and $y$. This within-sibship distribution is assumed to be normal with the mean as a linear function of the parental genotypic values $((x+y) / 2$, in the case of sex-independent characters). A controversy (recently reflected in the debate between Felsenstein (1981) and Feldman and Cavalli-Sforza (1981)) has arisen around the assumptions made in existing models concerning the within-subship variance. There are two aspects of this problem. First, it is always assumed that in a given generation the within-sibship variance is the same for all sibships, independent of their parents. In spite of being quite unrealistic, this assumption has not been subject to debate, although it would seem more realistic to expect the variance within a sibship to depend on the parental genotypic values, so that, for example, the variance within a sibship where both parents have either a maximal or a minimal genotypic value is less as compared with the variance within a subship where both parents have intermediate genotypic values. To support the assumption of the same for all sibships variance, Slatkin and Lande (1976) argued that in a natural 
population individuals with an extreme genotypic value are rare. It is not clear, however, how "extreme" the individuals ought to be in order for the differences between variances within different sibships to be important. And, in any case, this argument can be valid only provided that the evolutionary forces affecting the population dynamics are considered during a short-time period. It may also be argued that for some models, if the actual variances within individual sibships have been replaced by their average over all parental pairs, the new model, while obviously having the same variance within all sibships, will at the same time be mathematically equivalent to the original model. However, this is not always true (it is definitely not true for such essentially nonlinear models as those dealing with selection) and furthermore, the average value of the variances cannot usually be determined unless the actual variances within individual sibships are known in the first place. This brings up the second aspect of the problem of the within-sibship variance, the one causing debates: given that the variance is assumed to be the same within all sibships, what value should be assigned to it? Two kinds are distinguished among the existing models in this aspect. It is assumed in some that the within-sibship variance in a given generation takes the value equal to one half of the variance in the parental population in this generation. In other models the within-sibship variance is assumed to be a value remaining constant from generation to generation. This value is interpreted by some authors as one half of the variance in the population at linkage equilibrium, whereas others view it as just a constant parameter accounting for hereditary and developmental processes.

Thus, the distribution of the genotypic values (or phenotypes, in the case of phenotypic models) within any sibship is assumed in the existing models as normal with the mean (for sex-independent characters) equal to the midparental value, and with the variance equal to either one half of the variance among the parents or just a constant. A distinctive feature of these models is that they have very few parameters. In fact, the models with the within-sibship variance as one half of the variance among the parents have no free parameters at all, whereas models with the within-sibship variance as a constant have only one free parameter. Having very few parameters renders these models obvious advantages in comparison with the genic ones: they are relatively simple analytically and computationally, and it is possible for their few parameters to be sufficiently accurately estimated for a real quantitative character from experiments and observations. There are, however, certain disadvantages. If a model of a quantitative character dynamics does not have free parameters (as is the case where the withinsibship variance is one half of the variance among the parents), then, according to this model, given a set of evolutionary factors, all quantitative characters have the same dynamics, regardless of their hereditary and developmental properties_-an apparently unrealistic assertion. On the other 
hand, models with the within-sibship variance as a constant do not possess any genetic specificity and are, in fact, so general that they may as well be regarded as models of cultural or another form of heredity. This was clearly demonstrated in works by Karlin (e.g., Karlin, 1979a) where "cultural and biological variables are handled in one framework." Another problem with models of this kind is that it is very difficult to justify how the withinsibship variance, which must depend on the genetic structure of the population, can remain constant, especially if mechanisms such as crossing-over are to be accounted for by the model.

It thus appears that the applicability of existing zygotic/phenotypic models is restricted mainly to situations where the genetic structure of a population does not change substantially. This means that these models may be useful in predicting short-term dynamics of quantitative characters, provided that the evolutionary factors affecting the dynamics are sufficiently weak. At the same time, the significance of general inferences about longterm effects of different evolutionary factors on quantitative characters derived from the existing zygotic/phenotypic models is doubtful.

This brief discussion of the genic and zygotic/phenotypic models leads one to conclude that there is a definite gap between the two types. The zygotic/phenotypic "macro" models have actually no connections with the genic "micro" models (although an attempt has been made by Rocklin and Oster (1976) to derive a phenotypic model from a genic one). It is not clear whether and under what conditions the zygotic/phenotypic models may be considered as approximations of the genic ones. A substantial gap between the spheres of the models' applicability is also apparent, with the zygotic/phenotypic models being appropriate for predicting short-term dynamics, and with the genic ones being useful for investigating general evolutionary aspects of quantitative character dynamics.

An attempt is made in this paper to fill the gap between genic and zygotic/phenotypic models by introducing another "macro" type of models which may be called "gametic." Dealing with the gamete as the elementary unit of evolution, models of this type, unlike the zygotic/phenotypic, do incorporate some of the genetic mechanisms of quantitative characters (such as the gametic segregation and crossing-over) although in a more generalized form than in genic models. It is hoped that gametic models, while combining advantages of both genic and zygotic/phenotypic ones, will not pick up too many of their drawbacks. Gametic models may predict actual dynamics of quantitative characters more accurately and during longer periods of time than do the zygotic/phenotypic models. At the same time, they may be more efficient (although less precise) than the genic ones for obtaining some general theoretical results about relatively long-term effects on quantitative characters of different evolutionary forces. 


\section{THE MODEL}

In keeping with the traditions of population genetics, the value $X$ of a quantitative character for an individual is assumed to be a sum of two components:

$$
X=x+e,
$$

where $x$ is the component due to the individual's genotype (often called the individual's genotypic value); and $e$ is the component due to environment. The environmental component will be assumed to be distributed independently of the genotypic value with mean 0 and variance $v_{\mathrm{e}}$.

Almost every eucaryotic organism begins as a fusion of gametes produced by its parents. Let us assume that some numerical value (gametic effect) is ascribed to every gamete, and that the genotypic value of an individual is a function of the gametic effects of the gametes constituting the individual's zygote:

$$
x=f(\alpha, \beta),
$$

where $\alpha$ and $\beta$ are the gametic effects. (Lowercase Greek letters will be used for gametic effects, lowercase Latin letters for genotypic values, and capital Latin letters for quantitative character values.) The function $f(\alpha, \beta)$, which will be called the developmental function, is supposed to account in a generalized form for all processes leading an organism from the fertilized egg to the stage where the quantitative character of interest is manifested.

When describing the dynamics of a quantitative character in populations, not only the developmental processes must be accounted for, but the hereditary processes as well. The main process determining heredity in eucaryotic organisms is that of the production of gametes or gametogenesis. There is no need within the frame of the gametic model to go into all the details of mechanisms of gametogenesis. It suffices to postulate the existence of some function $H(\lambda \mid \alpha, \beta)$, which will be called the gametogenetic function, that specifies the conditional probability for a gamete with effect $\lambda$ to be among all the gametes produces by an individual whose genotype is formed by gametes with effects $\alpha$ and $\beta$. The gametogenetic function is supposed to account in a generalized form for all mechanisms of gametogenesis, such as the gametic segregation, crossing-over, and mutations. Not all of these mechanisms are, of course, of equal importance in affecting the dynamics of a quantitative character in order to be accounted for by the gametogenetic function under different circumstances. Thus, for example, if the dynamics are considered only during a relatively short evolutionary time, mutations do not usually play an important role and need not be accounted for by this function. 
Suppose a quantitative character is sex-independent, i.e., all hereditary and developmental processes are the same in both sexes. Then the population structure may be characterized by the distribution $P_{k}(\alpha, \beta)$ of genotypes, expressed in terms of gametic effects, among individuals in generation $k$. The dynamics of the population structure is easily derived by using the gametogenetic function:

$$
\begin{aligned}
P_{k+1}(\lambda, \eta)= & \frac{1}{W_{k}} \int P_{k}(\alpha, \beta) Q_{k}(\gamma, \delta \mid \alpha, \beta) \\
& \times S(\alpha, \beta ; \gamma, \delta) H(\lambda \mid \alpha, \beta) H(\eta \mid \gamma, \delta) d(\alpha \beta \gamma \delta),
\end{aligned}
$$

where $W_{k}=\int P_{k}(\alpha, \beta) Q_{k}(\gamma, \delta \mid \alpha, \beta) S(\alpha, \beta ; \gamma, \delta) d(\alpha \beta \gamma \delta)$. The integration here is over variables $\alpha, \beta, \gamma$, and $\delta$; and $d(\alpha \beta \gamma \delta)$ replaces the usual $d \alpha d \beta d \gamma d \delta$ for notational brevity. $Q_{k}(\gamma, \delta \mid \alpha, \beta)$ in (3) is the conditional probability that an individual with genotype $(\gamma, \delta)$ mates in generation $k$ with an individual whose genotype is $(\alpha, \beta)$. This function accounts for all deviations from random mating that can take place in populations. In the case of random mating,

$$
Q_{k}(\gamma, \delta \mid \alpha, \beta)=P_{k}(\gamma, \delta)
$$

Selection of any kind is accounted for by the fitness function $S(\alpha, \beta ; \gamma, \delta)$ defined on the set of the genotypic pairs. If selection operates exclusively on individuals, i.e., there is no pair selection, this function is represented by a product of the individual fitnesses:

$$
S(\alpha, \beta ; \gamma, \delta)=s(\alpha, \beta) s(\gamma, \delta) .
$$

If the developmental function and the distribution of the environmental component are known, the distrubution of the quantitative character in any generation can, at least hypothetically, be computed from the distribution $P_{k}(\alpha, \beta)$. In practice, however, the interest is not as much in the dynamics of the entire distribution of a quantitative character as in the dynamics of its mean and variance. These are easily calculated from (1) if the mean and variance of the genotypic values and environmental component are known:

$$
\begin{aligned}
M_{k}^{P} & =M_{k}, \\
V_{k}^{P} & =V_{k}+v_{\mathrm{e}} .
\end{aligned}
$$

Here, $\boldsymbol{M}_{k}^{p}$ and $V_{k}^{p}$ are the mean and variance of a quantitative character, $M_{k}$ and $V_{k}$ are the mean and variance of the genotypic values, and $v_{\mathrm{e}}$ is the 
variance of the environmental component. The mean and variance of the genotypic value in any generation can be computed from the relationships

$$
\begin{aligned}
M_{k} & =\int f(\alpha, \beta) P_{k}(\alpha, \beta) d(\alpha \beta), \\
V_{k} & =\int f^{2}(\alpha, \beta) P_{k}(\alpha, \beta) d(\alpha \beta)-M_{k}^{2} .
\end{aligned}
$$

Expression (3) may be viewed as a general gametic model of the quantitative character dynamics. The unrestricted generality of the functions entering this expression, however, prevents the model from being treated analytically or even numerically, and some assumptions restricting the generality of functions $f(\alpha, \beta)$ and $H(\lambda \mid \alpha, \beta)$ are needed. It may be assumed, for example, that they belong to some specified classes of functions. How should these classes be specified? It would be ideal, of course, to base the specification on what is known about the processes of quantitative character development and heredity. Unfortunately, what is known (or rather unknown) about these processes does not provide much insight about the classes to which the developmental and gametogenetic functions for real quantitative characters may belong. The only thing that can be stated with certainty is that these functions may be very complex and quite different for different characters and organisms. With such a lack of knowledge and insight, the only option left for restricting their generality is to approximate these functions by some mathematical expressions that are convenient for analytical or numerical manipulations. Even if regarded merely as a mathematical trick, such an approximation will have the virtue of making it possible to obtain some conclusions about the dynamics of quantitative characters (although not very precise) which otherwise would not be obtainable.

An approximation by a bivariate polynomial can be suggested for the developmental function, as an example. The genotypic value of an individual can then be represented as a first approximation by just the sum of the gametic effects of the gametes constituting the individual's genotype:

$$
f(\alpha, \beta)=\alpha+\beta .
$$

This presentation is not unusual in population genetics, where it holds true for all so-called additive characters. Also, it may hold as a good approximation for nonadditive character as well, if dominant as well as epistatic effects in different loci act in opposite direction, balancing each other (J. Crow, personal communication). If, however, this approximation is not satisfactory, the next polynomial term can be added:

$$
f(\alpha, \beta)=A(\alpha+\beta)+D \alpha \beta,
$$

thus reflecting a "gametic interaction." 
Assuming the developmental function in the form (8) as a first approximation, the mean and variance of the genotypic value in generation $k+1$ are obtained, using $(3)$ and $(7 \mathrm{a}, \mathrm{b})$, as

$$
\begin{aligned}
M_{k+1}= & \int(\lambda+\eta) P_{k+1}(\lambda, \eta) d(\lambda \eta) \\
= & \frac{1}{W_{k}} \int P_{k}(\alpha, \beta) Q_{k}(\gamma, \delta \mid \alpha, \beta) S(\alpha, \beta ; \gamma, \delta) \\
& \times\left[m_{H}(\alpha, \beta)+m_{H}(\gamma, \delta)\right] d(\alpha \beta \gamma \delta), \\
V_{k+1}= & \int(\lambda+\eta)^{2} P_{k+1}(\lambda, \eta) d(\lambda \eta)-M_{k+1}^{2} \\
= & \frac{1}{W_{k}} \int P_{k}(\alpha, \beta) Q(\gamma, \delta \mid \alpha, \beta) S(\alpha, \beta ; \gamma, \delta)\left[m_{H}^{\prime \prime}(\alpha, \beta)+m_{H}^{\prime \prime}(\gamma, \delta)\right. \\
& \left.+2 m_{H}(\alpha, \beta) m_{H}(\gamma, \delta)\right] d(\alpha \beta \gamma \delta)-M_{k+1}^{2} .
\end{aligned}
$$

Here, $m_{H}(\alpha, \beta)$ and $m_{H}^{\prime \prime}(\alpha, \beta)$ are the first and second moments of the gametogenetic function:

$$
\begin{aligned}
& m_{H}(\alpha, \beta)=\int \lambda H(\lambda \mid \alpha, \beta) d \lambda, \\
& m_{H}^{\prime \prime}(\alpha, \beta)=\int \lambda^{2} H(\lambda \mid \alpha, \beta) d \lambda
\end{aligned}
$$

Hence, if the developmental function is approximated as in (8), it is necessary to know only the first and second moments of the gametogenetic function to determine the genotypic value mean and variance in the population in the next generation. It is not at all clear what kind of a function the moments $m_{H}(\alpha, \beta)$ and $m_{H}^{\prime \prime}(\alpha, \beta)$ can be for real quantitative characters. Thereforc, lct us resort, as before, to a mathematical approximation of these functions by some bivariate polynomials, preferably of a minimal degree. A reasonable minimal degree of a polynomial to approximate $m_{H}(\alpha, \beta)$ should be 1 . Then, the minimal degree of a polynomial for $m_{H}^{\prime \prime}(\alpha, \beta)$ cannot, of course, be less than 2 . Thus, as a first approximation, the first and second moments of a gametogenetic function may be represented as

$$
\begin{aligned}
& m_{H}(\alpha, \beta)=b_{1}(\alpha+\beta)+b_{0}, \\
& m_{H}^{\prime \prime}(\alpha, \beta)=a_{3}\left(\alpha^{2}+\beta^{2}\right)+a_{2} \alpha \beta+a_{1}(\alpha+\beta)+a_{0} .
\end{aligned}
$$

If for some reason this approximation is not satisfactory, the next terms of the polynomial expansions can be considered. 
The substitution of (11a) and (11b) into (9a) and (9b) yields

$$
\begin{aligned}
M_{k+1}= & \frac{2}{W_{k}} \int\left[b_{1}(\alpha+\beta)+b_{0}\right] P_{k}(\alpha, \beta) Q_{k}(\gamma, \delta \mid \alpha, \beta) S(\alpha, \beta ; \gamma, \delta) d(\alpha \beta \gamma \delta), \\
V_{k+1}= & \frac{2}{W_{k}} \int\left[a_{3}\left(\alpha^{2}+\beta^{2}\right)+a_{2} \alpha \beta+\left(a_{1}+2 b_{1} b_{0}\right)(\alpha+\beta)+\left(a_{9}+b_{0}^{2}\right)\right. \\
& \left.+b_{1}^{2}(\alpha+\beta)(\gamma+\delta)\right] P_{k}(\alpha, \beta) Q_{k}(\gamma, \delta \mid \alpha, \beta) S(\alpha, \beta ; \gamma, \delta) \\
& \times d(\alpha \beta \gamma \delta)-M_{k+1}^{2} .
\end{aligned}
$$

These relations together with $(6 a)$ and $(6 b)$ provide a mathematical model that can be used to investigate approximate dynamics of the mean and variance of sex-independent quantitative characters in populations under various evolutionary factors.

Besides the distribution of genotypes, the distribution of the gametic effects,

$$
p_{k}(\alpha)=\int P_{k}(\alpha, \beta) d \beta
$$

may be of interest. When mating is random and selection is individual, the dynamics of the gametic effect distribution for $k>0$ is easily derived from (3) by integrating its both sides over variable $\eta$ :

$$
p_{k+1}(\lambda)=\frac{1}{w_{k}} \int p_{k}(\alpha) p_{k}(\beta) s(\alpha, \beta) H(\lambda \mid \alpha, \beta) d(\alpha \beta),
$$

where $w_{k}=\int p_{k}(\alpha) p_{k}(\beta) s(\alpha, \beta) d(\alpha \beta)$. The expressions for the mean and variance of the gametic effects are also easily derived in this case:

$$
\begin{aligned}
m_{k+1}= & \frac{1}{w_{k}} \int\left(2 b_{1} \alpha+b_{0}\right) p_{k}(\alpha) p_{k}(\beta) s(\alpha, \beta) d(\alpha \beta), \\
v_{k+1}= & \frac{1}{w_{k}} \int\left(2 a_{3} \alpha^{2}+a_{2} \alpha \beta+2 a_{1} \alpha+a_{0}\right) \\
& \times p_{k}(\alpha) p_{k}(\beta) s(\alpha, \beta) d(\alpha \beta)-m_{k+1}^{2},
\end{aligned}
$$

and the simple relationships exist in this case between the mean and variance of the distribution of the genotypic values and those of the distribution of the gametic effects:

$$
\begin{aligned}
M_{k} & =2 m_{k}, \\
V_{k} & =2 v_{k} .
\end{aligned}
$$




\section{The Model for Sex-Dependent Characters}

It is not difficult to see how the model can be extended to incorporate sexdependent characters. Consider first a sex-controlled character, in which case both sexes may have similar genotypes, but the phenotypes of males and females are different. Let $P_{k}^{*}(\alpha, \beta)$ be the distribution of genotypes, expressed in terms of gametic effects, among males in generation $k$, and $P_{k}^{* *}(\alpha, \beta)$ be the distribution of the genotypes among females. Expressions for these distributions in the next generation can be presented as

$$
\begin{aligned}
P_{k+1}^{*}(\lambda, \eta)= & \frac{1}{W_{k}} \int P_{k}^{*}(\alpha, \beta) Q_{k}^{* *}(\gamma, \delta \mid \alpha, \beta) S(\alpha, \beta ; \gamma, \delta) \\
& \times H^{*}(\lambda \mid \alpha, \beta) H^{* *}(\eta \mid \gamma, \delta) d(\alpha \beta \gamma \delta), \\
P_{k+1}^{* *}(\lambda, \eta)= & \frac{1}{W_{k}} \int P_{k}^{*}(\alpha, \beta) Q_{k}^{* *}(\gamma, \delta \mid \alpha, \beta) S(\alpha, \beta ; \gamma, \delta) \\
& \times H^{*}(\lambda \mid \alpha, \beta) H^{* *}(\eta \mid \gamma, \delta) d(\alpha \beta \gamma \delta),
\end{aligned}
$$

where $W_{k}=\int P_{k}^{*}(\alpha, \beta) Q_{k}^{* *}(\gamma, \delta \mid \alpha, \beta) S(\alpha, \beta ; \gamma, \delta) d(\alpha \beta \gamma \delta)$. The meaning of the functions entering the above expressions is the same as of those entering (3), but in this case they become sex-dependent. Thus, $Q_{k}^{* *}(\gamma, \delta \mid \alpha, \beta)$ is the probability that a female has genotype $(\gamma, \delta)$, given that she has mated with a male whose genotype is $(\alpha, \beta)$. The gametogenetic function for males, $H^{*}(\lambda \mid \alpha, \beta)$ can be different from the gametogenetic function for females, $H^{* *}(\lambda \mid \alpha, \beta)$, as it will, for example, be in the case when crossing-over in males is different from crossing-over in females. The fitness function, $S(\alpha, \beta ; \gamma, \delta)$ in this case is not necessarily invariant with respect to the permutation of the pairs of variables $\alpha, \beta$ and $\gamma, \delta$, i.e., $S(\alpha, \beta ; \gamma, \delta) \neq S(\gamma, \delta ; \alpha, \beta)$; and if the selection is individual, then

$$
S(\alpha, \beta ; \gamma, \delta)=s^{*}(\alpha, \beta) s^{* *}(\gamma, \delta),
$$

where $s^{*}(\alpha, \beta)$ and $s^{* *}(\gamma, \delta)$ are the individual fitnesses of males and females, respectively.

Given the genotype of an individual, the phenotype in the case of sexcontrolled characters depends on the individual's sex:

$$
\begin{aligned}
& Y=f^{* *}(\alpha, \beta)+e^{*} \quad \text { (for males), } \\
& X=f^{* *}(\alpha, \beta)+e^{* *} \quad \text { (for females). }
\end{aligned}
$$

Here, $e^{*}$ and $e^{* *}$ are the environmental components for males and females, and $f^{*}(\alpha, \beta)$ and $f^{* *}(\alpha, \beta)$ are the developmental functions determining the 
genotypic values of an individual whose genotype is $(\alpha, \beta)$, given that it is a male or a female respectively. As a first approximation, the male and female developmental functions can be represented as

$$
\begin{aligned}
f^{*}(\alpha, \beta) & =\alpha+\beta, \\
f^{* *}(\alpha, \beta) & =C(\alpha+\beta)+B,
\end{aligned}
$$

thus making it possible to incorporate "multiplicative" (by means of the parameter $C$ ) as well as "additive" (by means of the parameter $B$ ) influence of sex on a quantitative character.

It follows from $(16 \mathrm{a}, \mathrm{b})$ that in the case of sex-controlled characters, the distribution of the genotypes is the same in both sexes after one generation, i.e.,

$$
P_{k}^{*}(\alpha, \beta)=P_{k}^{* *}(\alpha, \beta)=P_{k}(\alpha, \beta) \quad(\text { for } k \geqslant 1),
$$

and either one of the equations (16a) or (16b) is sufficient to describe the dynamics of the genotypic structure of a population:

$$
\begin{aligned}
P_{k+1}(\lambda, \eta)= & \frac{1}{W_{k}} \int P_{k}(\alpha, \beta) Q_{k}(\gamma, \delta \mid \alpha, \beta) S(\alpha, \beta ; \gamma, \delta) \\
& \times H^{*}(\lambda \mid \alpha, \beta) H^{* *}(\eta \mid \gamma, \delta) d(\alpha \beta \gamma \delta) .
\end{aligned}
$$

Introducing the first and second moments of the gametogenetic functions for males and females, and approximating them by bivariate polynomials as in $(11 a, b)$,

$$
\begin{aligned}
m_{*}(\alpha, \beta) & =\int \lambda H^{*}(\lambda \mid \alpha, \beta) d \lambda=b_{1}^{*}(\alpha+\beta)+b_{0}^{*} \\
m_{*}^{\prime \prime}(\alpha, \beta) & =\int \lambda^{2} H^{*}(\lambda \mid \alpha, \beta) d \lambda=a_{3}^{*}\left(a^{2}+\beta^{2}\right)+a_{2}^{*} \alpha \beta+a_{1}^{*}(\alpha+\beta)+a_{0}^{*} \\
m_{* *}(\alpha, \beta) & =\int \lambda H^{* *}(\lambda \mid \alpha, \beta) d \lambda=b_{1}^{* *}(\alpha+\beta)-b_{0}^{* *} \\
m_{* *}^{\prime \prime}(\alpha, \beta) & =\int \lambda^{2} H^{* *}(\lambda \mid \alpha, \beta) d \lambda \\
& =a_{3}^{* *}\left(\alpha^{2}+\beta^{2}\right)+a_{2}^{* *} \alpha \beta+a_{1}^{* *}(\alpha+\beta) a_{0}^{* *}
\end{aligned}
$$

the following expressions for the mean $M_{k+1}^{*}$ and variance $V_{k+1}^{*}$ of the 
genotypic values among males for a sex-controlled character emerge, given that the developmental function for males is in the form (19a):

$$
\begin{aligned}
M_{k+1}^{*}= & \frac{1}{W_{k}} \int\left[b_{1}^{*}(\alpha+\beta)+b_{1}^{* *}(\gamma+\delta)+b_{0}^{*}+b_{0}^{* *}\right] \\
& \times P_{k}(\alpha, \beta) Q_{k}(\gamma, \delta \mid \alpha, \beta) S(\alpha, \beta ; \gamma, \delta) d(\alpha \beta \gamma \delta), \\
V_{k+1}^{*}= & \frac{1}{W_{k}} \int\left[a_{3}^{*}\left(\alpha^{2}+\beta^{2}\right)+a_{3}^{* *}\left(\gamma^{2}+\delta^{2}\right)+a_{2}^{*} \alpha \beta+a_{2}^{* *} \gamma \delta\right. \\
& +\left(a_{1}^{*}+2 b_{1}^{*} b_{0}^{* *}\right)(\alpha+\beta)+\left(a_{1}^{* *}+2 b_{1}^{* *} b_{0}^{*}\right)(\gamma+\delta) \\
& +a_{0}^{*}+a_{0}^{* *}+2 b_{0}^{*} b_{0}^{* *} \\
& \left.+2 b_{1}^{*} b_{1}^{* *}(\alpha+\beta)(\gamma+\delta)\right] P_{k}(\alpha, \beta) Q_{k}(\gamma, \delta \mid \alpha, \beta) S(\alpha, \beta ; \gamma, \delta) \\
& \times d(\alpha \beta \gamma \delta)-\left(M_{k+1}^{*}\right)^{2} .
\end{aligned}
$$

If the developmental function for females is in the form (19b), the mean and variance of the genotypic values among females in any generation can be computed through those of males as

$$
\begin{aligned}
M_{k}^{* *} & =C M_{k}^{*}+B, \\
V_{k}^{* *} & =C^{2} V_{k}^{*} .
\end{aligned}
$$

Notice that for a sex-dependent character the order of the variables in $P_{k}(\alpha, \beta)$ is relevant, i.e., $P_{k}(\alpha, \beta) \neq P_{k}(\beta, \alpha)$, and two different distributions of gametic effects need to be introduced:

$$
p_{k}^{*}(\alpha)=\int P_{k}(\alpha, \beta) d \beta
$$

for the distribution of the gametic effects among the gametes received by individuals from their fathers, and

$$
p_{k}^{* *}(\alpha)=\int P_{k}(\beta, \alpha) d \beta
$$

for the distribution of the gametic effects among the gametes received by the individuals from their mothers. Relations describing the dynamics of the distributions of the gametic effects can be obtained by integrating (20) over $\eta$ for the gametes received from males, and over $\lambda$ for the gametes received 
from females. Given (21a, b) and (22a, b), the dynamics of the means and variances of the gametic effects in the case of random mating and individual selection, i.e., when

$$
\begin{aligned}
Q_{k}(\gamma, \delta \mid \alpha, \beta) & =P_{k}(\gamma, \delta), \\
S(\alpha, \beta ; \gamma, \delta) & =s^{*}(\alpha, \beta) s^{* *}(\gamma, \delta),
\end{aligned}
$$

are described by the relations

$$
\begin{aligned}
m_{k+1}^{*}= & \frac{1}{w_{k}^{*}} \int\left[b_{1}^{*}(\alpha+\beta)+b_{0}^{*}\right] p_{k}^{*}(\alpha) p_{k}^{* *}(\beta) s^{*}(\alpha, \beta) d(\alpha \beta), \\
v_{k+1}^{*}= & \frac{1}{w_{k}^{*}} \int\left[a_{3}^{*}\left(\alpha^{2}+\beta^{2}\right)+a_{2}^{*} \alpha \beta+a_{1}^{*}(\alpha+\beta)+a_{0}^{*}\right] \\
& \times p_{k}^{*}(\alpha) p_{k}^{* *}(\beta) s^{*}(\alpha, \beta) d(\alpha \beta)-\left(m_{k+1}^{*}\right)^{2},
\end{aligned}
$$

where $w_{k}^{*}=\int p_{k}^{*}(\alpha) p_{k}^{* *}(\beta) s^{*}(\alpha, \beta) d(\alpha \beta)$;

$$
\begin{aligned}
m_{k+1}^{* *}= & \frac{1}{w_{k}^{* *}} \int\left[b_{1}^{* *}(\alpha+\beta)+b_{0}^{* *}\right] p_{k}^{* *}(\beta) s^{* *}(\alpha, \beta) d(\alpha \beta), \\
v_{k+1}^{* *}= & \frac{1}{w_{k}^{* *}} \int\left[a_{3}^{* *}\left(\alpha^{2}+\beta^{2}\right)+a_{2}^{* *} \alpha \beta+a_{1}^{* *}(\alpha+\beta)+a_{0}^{* *}\right] \\
& \times p_{k}^{*}(\alpha) p_{k}^{* *}(\beta) s^{* *}(\alpha, \beta) d(\alpha \beta)-\left(m_{k+1}^{* *}\right)^{2}
\end{aligned}
$$

where $w_{k}^{* *}=\int p_{k}^{*}(\alpha) p_{k}^{* *}(\beta) s^{* *}(\alpha, \beta) d(\alpha \beta)$. And if the developmental functions are in the form (19a) and (19b), the following relationships exist between the means and variances of the genotypic values and those of the gametic effccts:

$$
\begin{aligned}
M_{k}^{*} & =m_{k}^{*}+m_{k}^{* *}, \\
V_{k}^{*} & =v_{k}^{*}+v_{k}^{* *}, \\
M_{k}^{* *} & =C\left(m_{k}^{*}+m_{k}^{*}\right)+B, \\
V_{k}^{* *} & =C^{2}\left(v_{k}^{*}+v_{k}^{* *}\right) .
\end{aligned}
$$

In the case of sex-linked characters, assuming that males are haploid for the sex-linked genes and females are diploid, the genotypic structure of a population can be described by the univariate distribution of the genotypes expressed in terms of the gametic effects among males, $P_{k}^{*}(\alpha)$ and the 
bivariate distribution of the genotypes among females, $P_{k}^{* *}(\alpha, \beta)$. The following dynamical relations for these distributions are apparent:

$$
\begin{aligned}
P_{k+1}^{*}(\lambda)= & \frac{1}{W_{k}} \int P_{k}^{*}(\alpha) Q_{k}^{* *}(\gamma, \delta \mid \alpha) S(\alpha ; \gamma, \delta) \\
& \times H^{* *}(\lambda \mid \gamma, \delta) d(\alpha \gamma \delta), \\
P_{k+1}^{* *}(\lambda, \eta)= & \frac{1}{W_{k}} \int P_{k}^{*}(\alpha) Q_{k}^{* *}(\gamma, \delta \mid \alpha) S(\alpha ; \gamma, \delta) \\
& \times H^{*}(\lambda \mid \alpha) H^{* *}(\eta \mid \gamma, \delta) d(\alpha \gamma \delta),
\end{aligned}
$$

where $W_{k}=\int P_{k}^{*}(\alpha) Q^{* *}(\gamma, \delta \mid \alpha) S(\alpha ; \gamma, \delta) d(\alpha \gamma \delta)$. The meaning of the functions entering the above expressions is evident from the previous discussions.

The phenotypes of different sexes for a sex-linked character can be determined in the following way:

$$
\begin{array}{ll}
Y=f^{*}(\alpha)+e^{*} & \text { (for males), } \\
X=f^{* *}(\alpha, \beta)+e^{* *} & \text { (for females), }
\end{array}
$$

where $f^{*}(\alpha)$ and $f^{* *}(\alpha, \beta)$ are the developmental functions of males and females, respectively, for which the following expressions can be suggested as a first approximation:

$$
\begin{aligned}
f^{*}(\alpha) & =\alpha, \\
f^{* *}(\alpha, \beta) & =C(\alpha+\beta)+B .
\end{aligned}
$$

Introducing the first and second moments of the gametogenetic functions and approximating them by polynomials:

$$
\begin{aligned}
m_{*}(\alpha) & =\int \lambda H^{*}(\lambda \mid \alpha) d \lambda=b_{1}^{*} \alpha+b_{0}^{*}, \\
m_{*}^{\prime \prime}(\alpha) & =\int \lambda^{2} H^{*}(\lambda \mid \alpha) d \lambda=a_{3}^{*} \alpha^{2}+a_{1}^{*} \alpha+a_{0}^{*}, \\
m_{* *}(\alpha, \beta) & =\int \lambda H^{* *}(\lambda \mid \alpha, \beta) d \lambda=b_{1}^{* *}(\alpha+\beta)+b_{0}^{* *}, \\
m_{* *}^{\prime \prime}(\alpha, \beta) & =\int \lambda^{2} H^{* *}(\lambda \mid \alpha, \beta) d \lambda \\
& =a_{3}^{* *}\left(\alpha^{2}+\beta^{2}\right)+a_{2}^{* *} \alpha \beta+a_{1}^{* *}(\alpha+\beta)+a_{0}^{* *},
\end{aligned}
$$


dynamical relations for the means and variances of the genotypic values among males and females are obtained from (34) and (35) as

$$
\begin{aligned}
M_{k+1}^{*}= & \frac{1}{W_{k}} \int\left[b_{1}^{* *}(\gamma+\delta)+b_{0}^{* *}\right] P_{k}^{*}(\alpha) Q_{k}^{* *}(\gamma, \delta \mid \alpha) S(\alpha ; \gamma, \delta) d(\alpha \gamma \delta) \\
V_{k+1}^{*}= & \frac{1}{W_{k}} \int\left[a_{3}^{* *}\left(\gamma^{2}+\delta^{2}\right)+a_{2}^{* *} \gamma \delta+a_{1}^{* *}(\gamma+\delta)+a_{0}^{* *}\right] \\
& \times P_{k}^{*}(\alpha) Q_{k}^{* *}(\gamma, \delta \mid \alpha) S(\alpha ; \gamma, \delta) d(\alpha \gamma \delta)-\left(M_{k+1}^{*}\right)^{2} \\
M_{k+1}^{* *}= & \frac{C}{W_{k}} \int\left[b_{1}^{*} \alpha+b_{1}^{* *}(\gamma+\delta)+b_{0}^{*}+b_{0}^{* *}\right] \\
& \times P_{k}^{*}(\alpha) Q_{k}^{* *}(\gamma, \delta \mid \alpha) S(\alpha ; \gamma, \delta) d(\alpha \gamma \delta)+B \\
V_{k+1}^{* *}= & \frac{C^{2}}{W_{k}} \int\left[a_{3}^{*} \alpha^{2}+a_{3}^{* *}\left(\gamma^{2}+\delta^{2}\right)+a_{2}^{* *} \gamma \delta+\left(a_{1}^{*}+2 b_{1}^{*} b_{0}^{* *}\right) \alpha\right. \\
& +\left(a_{1}^{* *}+2 b_{1}^{* *} b_{0}^{*}\right)(\gamma+\delta)+2 b_{1}^{*} b_{1}^{* *} \alpha(\gamma+\delta) \\
& \left.+\left(a_{0}^{*}+a_{0}^{* *}+2 b_{0}^{*} b_{0}^{* *}\right)\right] \\
& \times P_{k}^{*}(\alpha) Q_{k}^{*}(\gamma, \delta \mid \alpha) S(\alpha ; \gamma, \delta) d(\alpha \gamma \delta)-\left(M_{k+1}^{* *}\right)^{2} .
\end{aligned}
$$

Let $p_{k}^{*}(\varepsilon)$ and $p_{k}^{* *}(\varepsilon)$ be the distributions of the gametic effects among the gametes obtained by individuals from their fathers and from their mothers, respectively, with the means and variances: $m_{k}^{*}, v_{k}^{*}$ and $m_{k}^{* *}, v_{k}^{* *}$. It is not difficult to see that for $k>0$, these distributions are the same for individuals of both sexes. The dynamics of these distributions in the case of random mating and individual selection, i.e., when

$$
\begin{aligned}
Q_{k}^{* *}(\gamma, \delta \mid \alpha) & =P_{k}^{* *}(\gamma, \delta), \\
S(\alpha ; \gamma, \delta) & =s^{*}(\alpha) s^{* *}(\gamma, \delta),
\end{aligned}
$$

are described by expressions following from (34) and (35):

$$
p_{k+1}^{*}(\lambda)=\frac{1}{w_{k}^{*}} \int p_{k}^{* *}(\alpha) s^{*}(\alpha) H^{*}(\lambda \mid \alpha) d \alpha,
$$

where $w_{k}^{*}=\int p_{k}^{* *}(\alpha) s^{*}(\alpha) d \alpha$,

$$
p_{k+1}^{* *}=\frac{1}{w_{k}^{* *}} \int p_{k}^{*}(\alpha) p_{k}^{* *}(\beta) s^{* *}(\alpha, \beta) H^{* *}(\lambda \mid \alpha, \beta) d(\alpha \beta),
$$


where $w_{k}^{* *}=\int p_{k}^{*}(\alpha) p_{k}^{* *}(\beta) s^{* *}(\alpha, \beta) d(\alpha \beta)$. The expression for the dynamics of means and variances of the gametic effects under random mating are obtained from the above expressions:

$$
\begin{aligned}
m_{k+1}^{*}= & \frac{1}{w_{k}^{*}} \int\left(b_{1}^{*} \alpha+b_{0}^{*}\right) p_{k}^{* *}(\alpha) s^{*}(\alpha) d \alpha, \\
v_{k+1}^{*}= & \frac{1}{w_{k}^{*}} \int\left(a_{3}^{*} \alpha^{2}+\alpha_{1}^{*} \alpha+a_{0}^{*}\right) p_{k}^{* *}(\alpha) s^{*}(\alpha) d \alpha-\left(m_{k+1}^{*}\right)^{2}, \\
m_{k+1}^{* *}= & \frac{1}{w_{k}^{* *}} \int\left(b_{1}^{* *}(\alpha+\beta)+b_{0}^{* *}\right) p_{k}^{* *}(\beta) s^{* *}(\alpha, b) d(\alpha \beta), \\
v_{k+1}^{* *}= & \frac{1}{w_{k}^{* *}} \int\left(a_{3}^{* *}\left(\alpha^{2}+\beta^{2}\right)+a_{2}^{* *} \alpha \beta+a_{1}^{* *}(\alpha+\beta)+a_{0}^{* *}\right) \\
& \times p_{k}^{*}(\alpha) p_{k}^{* *}(\beta) s^{* *}(\alpha, \beta) d(\alpha \beta)-\left(m_{k+1}^{* *}\right)^{2} .
\end{aligned}
$$

Under the random mating, the distributions of the genotypes and those of the gametic effects are connected as

$$
\begin{aligned}
P_{k}^{*}(\alpha) & =p_{k}^{* *}(\alpha), \\
P_{k}^{* *}(\alpha, \beta) & =p_{k}^{*}(\alpha) p_{k}^{* *}(\beta),
\end{aligned}
$$

and, if the developmental functions are in the form $(37 \mathrm{a}, \mathrm{b})$, the means and variances of the genotypic values are expressed through the means and variances of the gametic effects as

$$
\begin{aligned}
M_{k}^{* *} & =m_{k}^{* *}, \\
V_{k}^{*} & =v_{k}^{* *}, \\
M_{k}^{* *} & =C\left(m_{k}^{*}+m_{k}^{* *}\right)+B, \\
V_{k}^{* *} & =C^{2}\left(v_{k}^{*}+v_{k}^{* *}\right) .
\end{aligned}
$$

\section{The Gametic Model of a Polygenic Additive Character}

A number of questions about the model represented by relations $(12 a, b)$ (or by their extensions incorporating sex-dependent characters) may arise. Are the formulas for the developmental function (8) and for the first and second moments of the gametogenetic function $(11 \mathrm{a}, \mathrm{b})$ nothing more than just arbitrary mathematical simplifications, or they do reflect reality, at least 
in some cases? Are there restrictions on the parameters of the model or can they take any values? What are the values of these parameters that one may expect for real quantitative characters? The best way to answer these questions would be to observe the dynamics of quantitative characters in different biological populations under various conditions and determine the parameters of the model by the usual methods of parameter estimation. Unfortunately, there is a lack of sufficiently extensive observations and experiments on quantitative character dynamics in real populations; therefore, it is not a practical way of making inferences about the model and its parameters. Another way is to take advantage of our knowledge of the genetic mechanisms of inheritance and development and try to make inferences based on properties of these mechanisms about the gametic model. One possibility along this way is to turn to the traditional in population genetics model of polygenic, additive characters.

Let us start with sex-independent characters. According to the polygenic hypothesis, a quantitative character is under control of many loci with similar effects on the character. Additivity implies that the genotypic value of an individual is the sum of the effects of all the loci controlling the character. For characters of this type, it is natural to define the gametic effect as the sum of the effects of all loci carried by the gamete. Then, the genotypic value of an individual will certainly be equal to the sum of the effects of the gametes constituting the individual's genotype, i.e., the developmental function is as in (8).

The gametogenetic function for diploid organisms ought to reflect the three main processes of gametogenesis: segregation, mutation, and recombination. In general, this function can be represented as

$$
H(\lambda \mid \alpha, \beta)=\int h(\lambda \mid v) \stackrel{H}{H}(v \mid \alpha, \beta) d v,
$$

where $h(\lambda \mid v)$ is the probability that a gamete of effect $v$ is converted to one of effect $\lambda$ by mutation. The function $\dot{H}(v \mid \alpha, \beta)$ accounts for segregation and recombination. It provides the conditional probability that a gamete has effect $v$, given that it is produced by segregation and recombination in a zygote formed by gametes with effects $\alpha$ and $\beta$.

Notice that when mutation and recombination are neglected (which is the case of one multiallelic locus), the gametogenetic function has the form

$$
\begin{array}{rlrl}
H(\lambda \mid \alpha, \beta)=\frac{1}{2} & & (\lambda=\alpha), \\
& =0 & & (\lambda \neq \alpha, \beta), \\
& =\frac{1}{2} & & (\lambda=\beta),
\end{array}
$$


and its first and second moments are

$$
\begin{aligned}
& m_{H}(\alpha, \beta)=\frac{1}{2}(\alpha+\beta), \\
& m_{H}^{\prime \prime}(\alpha, \beta)=\frac{1}{2}\left(\alpha^{2}+\beta^{2}\right) .
\end{aligned}
$$

Thus, in this case, the first and second moments of the gametogenetic function are indeed first- and second-degree polynomials with the coefficients: $b_{1}=\frac{1}{2}, b_{0}=0 ; a_{3}=\frac{1}{2}, a_{2}=a_{1}=a_{0}=0$.

With segregation and mutation, but without recombination, the substitution of function $\dot{H}$ in the form (48) into (47) yields gametogenetic function

$$
H(\lambda \mid \alpha, \beta)=\frac{1}{2}[h(\lambda \mid \alpha)+h(\lambda \mid \beta)] .
$$

Its first and second moments are

$$
\begin{aligned}
& m_{H}(\alpha, \beta)=\frac{1}{2}\left[m_{h}(\alpha)+m_{h}(\beta)\right], \\
& m_{H}^{\prime \prime}(\alpha, \beta)=\frac{1}{2}\left[m_{h}^{\prime \prime}(\alpha)+m_{h}^{\prime \prime}(\beta)\right],
\end{aligned}
$$

where $m_{h}(\xi)$ and ${ }_{h}^{\prime \prime}(\xi)$ are the first and second moments of the conditional distribution $h(\lambda \mid \xi)$. To obtain mathematical expressions for this distribution and its moments, further formalization of the genetic system is needed. Restricting consideration to diallelic cases, every gametotype can be viewed as a sequence of digits, say 0 and 1 , of some length $n$ equal to the number of the loci controlling the character. If these digits are regarded as the effects of individual loci, then the gametic effects can take values between 0 and $n$. Genotypic values of individuals arc in this casc between 0 and $2 n$.

This formalization of genetic systems of polygenic, additive characters is not uncommon, especially for simulations of the dynamics of quantitative characters in computers. Within this scheme, the mutational process can be represented as follows: every allele 1 within any locus can be converted into allele 0 with probability $\mu_{+}$independently of all other loci; and every allele 0 can be converted into allele 1 with probability $\mu_{-}$. The function $h(\lambda \mid \alpha)$ is then a convolution of two binomial distributions:

$$
h(\lambda \mid \alpha)=\sum_{j} B\left[j ; \alpha,\left(1-\mu_{+}\right)\right] B\left[(\lambda-j) ;(n-\alpha), \mu_{-}\right],
$$

where $B[a ; b, c]$ stands for the binomial probability of $a$ successes in a Bernoulli series of length $b$ with the probability of a success equal to $c$. For the moments of $h(\lambda \mid \alpha)$, it follows from (52) that

$$
\begin{aligned}
m_{h}(\alpha)= & \alpha\left(1-\mu_{+}-\mu_{-}\right)+n \mu_{-}, \\
m_{h}^{\prime \prime}(\alpha)= & \alpha^{2}\left(1-\mu_{+}-\mu_{-}\right)^{2}-\alpha\left(\mu_{+}\left(1-\mu_{+}\right)-\mu_{-}\left(1-\mu_{-}\right)\right. \\
& \left.+2 n \mu_{-}\left(1-\mu_{+}-\mu_{-}\right)\right)+n \mu_{-}\left(1-\mu_{-}+n \mu_{-}\right) .
\end{aligned}
$$


It is not difficult to show, using $(51 \mathrm{a}, \mathrm{b})$, that in this case, i.e., when only mutation and segregation are considered, conditions (11a) and (1 lb) hold. The first and second moments of the gametogenetic function are indeed firstand second-degree polynomials with coefficients

$$
\begin{gathered}
b_{1}=\frac{1}{2}\left(1-\mu_{+}-\mu_{-}\right), \quad b_{0}=n \mu_{-} ; \\
a_{3}=\frac{1}{2}\left(1-\mu_{+}-\mu_{-}\right)^{2}, \quad a_{2}=0 \\
a_{1}=\frac{1}{2}\left(1-\mu_{+}-\mu_{-}\right)\left(\mu_{+}-\mu_{-}\right)+n \mu_{-}\left(1-\mu_{+}-\mu_{-}\right), \\
a_{0}=n^{2} \mu_{-}^{2}+n \mu_{-}\left(1-\mu_{-}\right) .
\end{gathered}
$$

The main result of recombination is that a daughter gamete produced by a zygote receives some of its loci from one of the parental gametes constituting the zygote, and the rest of the loci from the other one. The gametic effect of the daughter gamete is, therefore, determined not only by the gametic effects of the parental gametes, but also by the arrangements of the allelic effects between the different loci in the parental gamete, i.e., by their gametotypes. Hence in general, the gametogenetic function should be represented as

$$
H(\lambda \mid \alpha, \beta)=\frac{\bigvee_{\alpha}}{\Upsilon_{\boldsymbol{\beta}}} \operatorname{Pr}[\lambda \mid \boldsymbol{\alpha}, \boldsymbol{\beta}] \operatorname{Pr}[\boldsymbol{\alpha}, \boldsymbol{\beta} \mid \alpha, \beta],
$$

where $\operatorname{Pr}[\lambda \mid \boldsymbol{\alpha}, \boldsymbol{\beta}]$ is the probability that a gamete of effect $\lambda$ is produced by a zygote formed by gametes with gametotypes $\boldsymbol{\alpha}$ and $\boldsymbol{\beta} . \operatorname{Pr}[\boldsymbol{\alpha}, \boldsymbol{\beta} \mid \alpha, \beta]$ is the probability that the gametes constituting a zygote are of gametotypes $\alpha$ and $\beta$, given that their gametic effects are $\alpha$ and $\beta$. The latter probability depends, in general, on the current genotypic structure of the population and thus may change from generation to generation. We shall assume, however, that

$$
\operatorname{Pr}[\boldsymbol{\alpha}, \boldsymbol{\beta} \mid \alpha, \beta]=\operatorname{Pr}[\boldsymbol{\alpha} \mid \alpha] \operatorname{Pr}[\boldsymbol{\beta} \mid \beta]
$$

in every generation, i.e., the probability for a gamete of a given effect in a zygote to be of a particular gametotype is independent of the other gamete in the zygote. This will be true, of course, in all cases when the evolutionary factors affecting the dynamics of a quantitative character act upon the phenotypes of individuals (e.g., in the case of phenotypic selection or phenotypic assortative mating), but not upon the individuals' genotypes. Under this assumption, the first and second moments of the gametogenetic function are

$$
\begin{aligned}
& m_{H}(\alpha, \beta)=\searrow_{\alpha} \searrow_{\boldsymbol{\beta}} E(\lambda \mid \boldsymbol{\alpha}, \boldsymbol{\beta}) \operatorname{Pr}[\boldsymbol{\alpha} \mid \alpha] \operatorname{Pr}[\boldsymbol{\beta} \mid \beta], \\
& m_{H}^{\prime \prime}(\alpha, \beta)=\frac{\coprod_{\alpha}}{V_{\boldsymbol{\beta}}} E\left(\lambda^{2} \mid \boldsymbol{\alpha}, \boldsymbol{\beta}\right) \operatorname{Pr}[\boldsymbol{\alpha} \mid \alpha] \operatorname{Pr}[\boldsymbol{\beta} \mid \beta],
\end{aligned}
$$


where $E()$ means the expected value. Assuming the character is polygenic, diallelic, and additive, and also employing the representation of gametotypes as sequences of digits 0 or 1 , the gametic effect of a gamete can be represented as

$$
\alpha=\sum_{i=1}^{n} \alpha_{i} \quad\left(\alpha_{i}=0,1\right)
$$

where $\alpha_{i}$ is the contribution of an individual locus, and $n$ is the number of loci controlling the character. Then,

$$
\begin{aligned}
E(\lambda \mid \boldsymbol{\alpha}, \boldsymbol{\beta}) & =E\left[\left(\lambda_{1}+\lambda_{2}+\cdots+\lambda_{n}\right) \mid \boldsymbol{\alpha}, \boldsymbol{\beta}\right], \\
E\left(\lambda^{2} \mid \boldsymbol{\alpha}, \boldsymbol{\beta}\right) & =E\left[\left(\lambda_{1}+\lambda_{2}+\cdots+\lambda_{n}\right)^{2} \mid \boldsymbol{\alpha}, \boldsymbol{\beta}\right] \\
& =E\left[\left(\frac{\searrow}{T} \lambda_{i}^{2}+\frac{\searrow}{i \neq j} \lambda_{i} \lambda_{j}\right) \mid \boldsymbol{\alpha}, \boldsymbol{\beta}\right] .
\end{aligned}
$$

If mutations are neglected, it follows form the Mendelian laws of segregation that

$$
\begin{aligned}
E(\lambda \mid \boldsymbol{\alpha}, \boldsymbol{\beta})= & \frac{1}{2} \sum_{i}\left(\alpha_{i}+\beta_{i}\right), \\
E\left(\lambda^{2} \mid \boldsymbol{\alpha}, \boldsymbol{\beta}\right)= & \frac{1}{2} \frac{\coprod_{i}}{i}\left(\alpha_{i}^{2}+\beta_{i}^{2}\right)+\frac{1}{2} \sum_{i \neq j} r_{i j}\left(\alpha_{i} \beta_{j}+\beta_{i} \alpha_{j}\right) \\
& +\frac{1}{2} \underset{i \neq j}{\bigvee}\left(1-r_{i j}\right)\left(\alpha_{i} \alpha_{j}+\beta_{i} \beta_{j}\right),
\end{aligned}
$$

where $r_{i j}$ is the probability of recombination between loci $i$ and $j$. Substituting these expressions into (57a) and (57b) yields, taking into consideration that the contributions of individual loci are either 0 or 1 ,

$$
\begin{aligned}
m_{H}(\alpha, \beta)= & \frac{1}{2} \sum_{i}\left(\operatorname{Pr}\left[\alpha_{i}=1 \mid \alpha\right]+\operatorname{Pr}\left[\beta_{i}=1|\beta|\right),\right. \\
m_{H}^{\prime \prime}(\alpha, \beta)= & \frac{1}{2} \sum_{i}\left(\operatorname{Pr}\left[\alpha_{i}=1 \mid \alpha\right]+\operatorname{Pr}\left[\beta_{i}=1|\beta|\right)\right. \\
& +\sum_{i \neq j} r_{i j} \operatorname{Pr}\left[\alpha_{i}=1 \mid \alpha\right] \operatorname{Pr}\left[\beta_{j}=1 \mid \beta\right] \\
& +\frac{1}{2} \sum_{i \neq j}\left(1-r_{i j}\right)\left(\operatorname{Pr}\left[\alpha_{i}=1, \alpha_{j}=1 \mid \alpha\right]\right. \\
& \left.+\operatorname{Pr}\left[\beta_{i}=1, \beta_{j}=1 \mid \beta\right]\right) .
\end{aligned}
$$


It is shown in the Appendix that

$$
\sum_{i} \operatorname{Pr}\left[\alpha_{i}=1 \mid \alpha\right]=\alpha
$$

Therefore,

$$
m_{H}(\alpha, \beta)=\frac{1}{2}(\alpha+\beta)
$$

i.e., not surprisingly, the first moment of the gametogenetic function for a polygenic, diallelic, additive character is a linear polynomial of the type (11a) with the coefficients $b_{1}=\frac{1}{2}, b_{0}=0$, regardless of recombination mechanism.

As for the second moment of the gametogenetic function, let us first consider the case when there is no recombination between loci, i.e., $r_{i j}=0$ for all $i$ and $j$. It follows from (62) that in this case,

$$
\begin{aligned}
m_{H}^{\prime \prime}(\alpha, \beta)= & \frac{1}{2} \frac{\backslash}{i}\left(\operatorname{Pr}\left[\alpha_{i}=1 \mid \alpha\right]+\operatorname{Pr}\left[\beta_{i}=1 \mid \beta\right]\right) \\
& \left.+\frac{1}{2} \sum_{i \neq j}\left(\operatorname{Pr}\left|\alpha_{i}-1, \alpha_{j}=1\right| \alpha\right]+\operatorname{Pr}\left|\beta_{i}=1, \beta_{j}=1\right| \beta \mid\right) .
\end{aligned}
$$

It is also shown in the Appendix that

$$
\sum_{i \neq j} \operatorname{Pr}\left[\alpha_{i}=1, \alpha_{j}=1 \mid \alpha\right]=\alpha(\alpha-1)
$$

The substitution of this and (63) in (65) yields

$$
m_{H}^{\prime \prime}(\alpha, \beta)=\frac{1}{2}(\alpha+\beta)+\frac{1}{2}[\alpha(\alpha-1)+\beta(\beta-1)]=\frac{1}{2}\left(\alpha^{2}+\beta^{2}\right),
$$

which is, of course, the same as (49b). Thus, in absence of recombination, the right side of expression (62) is a second-degree polynomial.

If there is a recombination between the loci controlling a quantitative character, expression (62) does not indicate anymore that $m_{H}^{\prime \prime}(\alpha, \beta)$ is necessarily a second-degree polynomial. Let us, however, take a different approach. Let $P(1 \mid \alpha)$ and $P(1,1 \mid \alpha)$ stand for the probabilities $\operatorname{Pr}\left|\alpha_{i}=1\right| \alpha \mid$ and $\operatorname{Pr}\left[\alpha_{i}=1, \alpha_{j}=1 \mid \alpha\right]$ averaged over all the $n$ loci of a gamete, i.e.,

$$
\begin{aligned}
P(1 \mid \alpha) & =\frac{1}{n} \sum_{i=1}^{n} \operatorname{Pr}\left[\alpha_{i}=1 \mid \alpha\right], \\
P(1,1 \mid \alpha) & =\frac{1}{n(n-1)} \sum_{i \neq j}^{n} \operatorname{Pr}\left[\alpha_{i}=1, \alpha_{j}=1 \mid \alpha\right] .
\end{aligned}
$$


It follows from (63) and (66) that

$$
\begin{aligned}
P(1 \mid \alpha) & =\alpha / n, \\
P(1,1 \mid \alpha) & =\alpha(\alpha-1) / n(n-1) .
\end{aligned}
$$

Let us now substitute these average values instead of the actual probabilities entering expression (62), thus yielding

$$
\begin{aligned}
m_{H}^{\prime \prime}(\alpha, \beta)= & \frac{1}{2}(\alpha+\beta)+\alpha \beta \underset{i \neq j}{\bigvee} r_{i j} / n^{2} \\
& +\frac{1}{2}[\alpha(\alpha-1)+\beta(\beta-1)] \sum_{i \neq j}^{\backslash}\left(1-r_{i j}\right) / n(n-1) .
\end{aligned}
$$

The substitution of the average values $P(1 \mid \alpha)$ and $P(1,1 \mid \alpha)$ instead of the actual probabilities is equivalent to making the assumption of exchangeability of the loci controlling the quantitiative character:

$$
\begin{aligned}
\operatorname{Pr}\left[\alpha_{i}=1 \mid \alpha\right] & =P(1 \mid \alpha) & & \text { (for all } i), \\
\operatorname{Pr}\left[\alpha_{i}=1, \alpha_{j}=1 \mid \alpha\right] & =P(1,1 \mid \alpha) & & (\text { for all } i \neq j) .
\end{aligned}
$$

By making this assumption, we have in essence replaced the traditional deterministic concept of a biological population with a statistical concept of a "population" as the average over the ensemble of all the deterministic populations having the same distribution of gametic effects, but with possibly different distributions of gametotypes.

Notice that if the allelic frequencies are the same in all the loci and there is free recombination between the loci, the probabilities $\operatorname{Pr}\left[\alpha_{i}=1|\alpha|\right.$ and $\operatorname{Pr}\left|\alpha_{i}=1, \alpha_{j}=1\right| \alpha \mid$ are the same for all $i$ and $j$ and coincide, obviously, with their average values $P(1 \mid \alpha)$ and $P(1,1 \mid \alpha)$. There is, therefore, no difference in this case between the "statistical" and "deterministic" approaches, and the gametic model with the first and second moments of the gametogenetic function given by

$$
\begin{aligned}
& m_{H}(\alpha, \beta)=\frac{1}{2}(\alpha+\beta), \\
& m_{H}^{\prime \prime}(\alpha, \beta)=\frac{1}{4}\left(\alpha^{2}+\beta^{2}\right)+\frac{n-1}{2 n} \alpha \beta+\frac{1}{4}(\alpha+\beta)
\end{aligned}
$$

is equivalent to the corresponding genic model.

Introducing the parameter

$$
R=\underset{i \neq j}{\Gamma} r_{i j} / 2 n^{2}
$$


expression (71) can be rewritten as

$$
m_{H}^{\prime \prime}(\alpha, \beta)=\left(\frac{1}{2}-\frac{n}{n-1} R\right)\left(\alpha^{2}+\beta^{2}\right)+2 R \alpha \beta+\frac{n}{n-1} R(\alpha+\beta) .
$$

It is not difficult to show that if the contributions of individual loci to a quantitative character are $\varepsilon_{0}$ and $\varepsilon_{1}$ instead of 0 and 1 , we have

$$
\begin{aligned}
m_{H}(\alpha, \beta)= & \frac{1}{2}(\alpha+\beta), \\
m_{H}^{\prime \prime}(\alpha, \beta)= & \left(\frac{1}{2}-\frac{n}{n-1} R\right)\left(\alpha^{2}+\beta^{2}\right)+2 R \alpha \beta+\frac{n}{n-1} R\left(\varepsilon_{1}+\varepsilon_{0}\right)(\alpha+\beta) \\
& -\frac{2 n^{2}}{n-1} R \varepsilon_{1} \varepsilon_{0} .
\end{aligned}
$$

Hence, in a population viewed as a statistical entity, the first and second moments of the gametogenetic function accounting for recombination and gametic segregation of a polygenic, diallelic, additive characters are firstand second-degree polynomials with the coefficients

$$
\begin{gathered}
b_{1}=\frac{1}{2}, \quad b_{0}=0 ; \\
a_{3}=\frac{1}{2}-\frac{n}{n-1} R, \quad a_{2}=2 R, \\
a_{1}=\frac{n}{n-1} R\left(\varepsilon_{1}+\varepsilon_{0}\right), \quad a_{0}=-\frac{2 n^{2}}{n-1} \varepsilon_{1} \varepsilon_{0} .
\end{gathered}
$$

Notice that only coefficients $a_{1}$ and $a_{0}$ depend on the actual values of allelic effects, whereas coefficients $a_{3}$ and $a_{2}$ remain the same whether the allelic effects are 0 and 1 or some other values. Thus, coefficients $a_{1}$ and $a_{0}$ serve as scale parameters of the gametic model, whereas the mechanisms of recombination and gametic segregation are accounted for in the model by the coefficients $a_{3}$ and $a_{2}$, or by only two parameters $n$ and $R$. If the number of loci is sufficiently large, so that $n /(n-1) \simeq 1$, there remains only one parameter $R$. Properties of this parameter are discussed in the Appendix, and Fig. 1 shows how it depends on the number of loci for different recombination rates. It is seen that the values of the parameter lie between 0 , when recombination is low and the number of loci is small, and $\frac{1}{4}$, when the number of loci increases to infinity.

When a gametic model is used to investigate the consequences of specific evolutionary factors, it may always be assumed that a character is measured on the scale corresponding to the allelic effects being as either 0 or 1 , so that only two of the coefficients (76b) are independent. When trying, however, to 


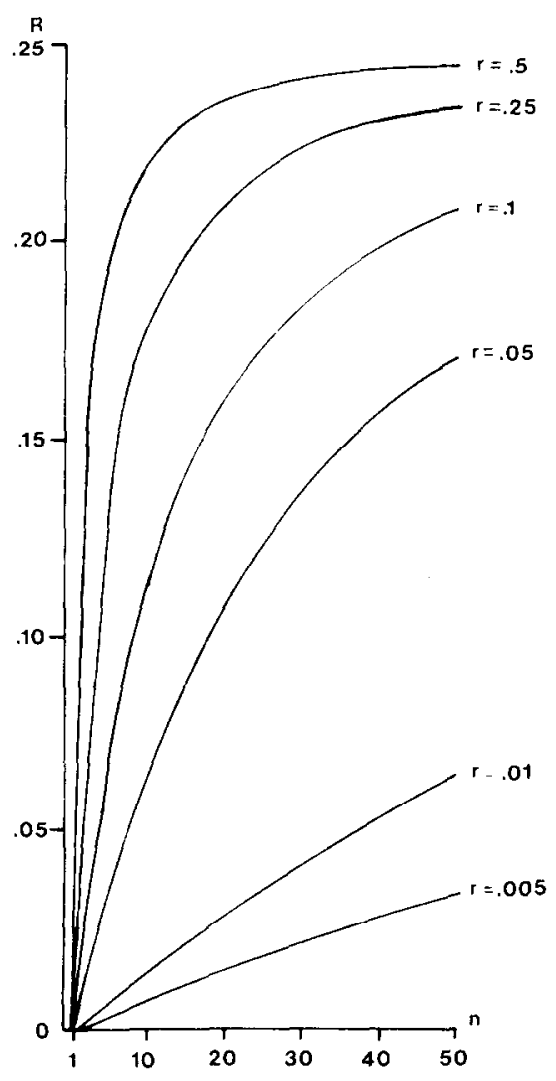

FIG. 1. Parameter $R$ as a function of number of loci $n$ and rate of recombination $r$.

apply the gametic model to predict the dynamics of a particular character in a natural population, there always will be a "natural" scale of the character measurement. Usually this scale will be determined by the units in which the evolutionary factor affecting the character dynamics is measured. Thus, for example, if selection acting in a population is measured in terms of the fitnesses ascribed to different values of the quantitative character, then the "natural" scale is determined by the units in which these values are measured (i.e., meters, centimeters, or inches for, say, height). The allelic effects will, of course, in this case be determined by the "natural" scale, and all four of the coefficients $(76 \mathrm{~b})$ must be assumed as mutually independent.

If the gametogenetic function of a polygenic character is supposed to account for all three main processes of gametogenesis: segregation, crossingover, and mutation, it follows from (47), (54a, b), and (74), (75) that its first 
and second moments are first- and second-degree polynomials with the coefficients

$$
\begin{gathered}
b_{1}=\frac{1}{2}\left(1-\mu_{+}-\mu_{-}\right), \quad b_{0}=n\left(\varepsilon_{0} \mu_{+}+\varepsilon_{1} \mu_{-}\right) \\
a_{3}=\left(\frac{1}{2}-\frac{n}{n-1} R\right)\left(1-\mu_{+}-\mu_{-}\right)^{2}, \quad a_{2}=2 R\left(1-\mu_{+}-\mu_{-}\right)^{2}, \\
a_{1}=\frac{n}{n-1} R\left(\varepsilon_{0}+\varepsilon_{1}\right)+\frac{1}{2}\left(\left(\varepsilon_{1}-\varepsilon_{0}\right)\left(\mu_{+}-\mu_{-}\right)\right. \\
\left.+2 n\left(\varepsilon_{0} \mu_{+}+\varepsilon_{1} \mu_{-}\right)\right)\left(1-\mu_{+}-\mu_{-}\right), \\
a_{0}=-\frac{2 n^{2}}{n-1} R \varepsilon_{1} \varepsilon_{0}+n\left(\varepsilon_{1}-\varepsilon_{0}\right)\left(\left(\varepsilon_{1} \mu_{-}\left(1-\mu_{-}\right)-\varepsilon_{0} \mu_{+}\left(1-\mu_{+}\right)\right) .\right.
\end{gathered}
$$

When the dynamics of a quantitative character are considered during a relatively small number of generations, the coefficients may always be assumed in the form $(76 \mathrm{a}, \mathrm{b})$, since the effects of mutations are usually very small in comparison with the effects of crossing-over and gametic segregation. If, however, the character dynamics are considered during a large number of generations, the mutational process cannot be ignored, and the coefficients given in $(77 \mathrm{a}, \mathrm{b})$ should be used.

Considering generalizations of the gametic model on sex-dependent, polygenic, diallelic, additive characters, it is reasonable to assume in all cases when mutations cannot be neglected, that the mutational processes are the same in males as in females. This assumption, which is close to what is observed in the majority of living organisms, will be made in all future discussions. In the case of sex-controlled characters, this will imply the following relationships between the coefficients of the first moments of the gametogenetic functions for males and females:

$$
b_{1}^{* *}=b_{1}^{*}=b_{1}, \quad b_{0}^{* *}=b_{0}^{*}=b_{0},
$$

where $b_{1}$ and $b_{0}$ are given in (77a). The parameter $R$ entering expressions (77b) for the coefficients of the second moments of the gametogenetic functions may in the case of sex-controlled characters have different values: $R^{*}$ for males and $R^{* *}$ for females, due to the possible differences in crossing-over between the sexes.

In the case of sex-linked characters, the gametogenetic function for males may be assumed as

$$
H^{*}(\lambda \mid \alpha)=h(\lambda \mid \alpha),
$$

where $h(\lambda \mid \alpha)$ is given by (52), with the first and second moments by (53a, b). The gametogenetic function for females, $H^{* *}(\lambda \mid \alpha, \beta)$, on the other hand, can be derived in the same way as before, and its first and second 
moments are first- and second-degree polynomials with the coefficients given by $(77 \mathrm{a}, \mathrm{b})$ with the parameter $R=R^{* *}$ determined by crossing-over in females. The assumption of the same mutational processes in both sexes implies in this case the following relationships between the coefficients of the first moment of the gametogenetic functions for males and for females:

$$
b_{1}^{* *}=\frac{1}{2} b_{1}^{*}=b_{1}, \quad b_{0}^{* *}=b_{0}^{*}=b_{0},
$$

with $b_{1}$ and $b_{0}$ given by $(77 \mathrm{a})$.

\section{Quantitative Character Dynamics under Random Mating}

In this section, the gametic model will be applied to the dynamics of the mean and variance of a quantitative character in the simplest case of a population under random mating without selection.

Let us first assume that the character is sex-independent. Assuming also an additive developmental function, i.e., (8), expressions (12a) and (12b) can be used to obtain the dynamical equations for the mean and variance of the genotypic values:

$$
\begin{aligned}
M_{k+1}= & 2 b_{1} M_{k}+2 b_{0}, \\
V_{k+1}= & 2 a_{3} V_{k}+\left(a_{3}+a_{2} / 2-2 b_{1}^{2}\right) M_{k}^{2}+2\left(a_{1}-2 b_{1} b_{0}\right) M_{k}+2\left(a_{0}-b_{0}\right) \\
& (\text { for } k \geqslant 1) .
\end{aligned}
$$

It is easy to see from these equations that when neither mutation nor recombination are present, so that the coefficients of the model are $b_{1}=\frac{1}{2}$, $b_{0}=0 ; a_{3}=\frac{1}{2}, a_{2}=a_{1}=a_{0}=0$, the genotypic mean and variance do not change from their values in the first generation. This is in agreement, of course, with the well-known result that a one-locus genetic system under random mating without selection is in Hardy-Weinberg equilibrium. In general, however, expressions $(80 a, b)$ constitute a system of recursive equations whose solution is

$$
\begin{aligned}
M_{k} & =C_{1}\left(2 b_{1}\right)^{k}+\hat{M}, \\
V_{k} & =C_{2}\left(2 a_{3}\right)^{k}+C_{3}\left(2 b_{1}\right)^{2 k}+C_{4}\left(2 b_{1}\right)^{k}+\hat{V},
\end{aligned}
$$

where $C_{i}$ are constants determined by the genotypic value mean and variance in the first generation; and $\hat{M}, \hat{V}$ are the equilibrium values

$$
\begin{aligned}
\hat{M} & =2 b_{0} /\left(1-2 b_{1}\right), \\
\hat{V} & =\left[\left(a_{3}+a_{2} / 2-2 b_{1}^{2}\right) \hat{M}^{2}+2\left(a_{1}-2 b_{1} b_{0}\right) \hat{M}+2\left(a_{0}-b_{0}^{2}\right)\right] /\left(1-2 a_{3}\right) .
\end{aligned}
$$


Equations for the dynamics and equilibrium values of the phenotypic mean and variance are obtained from $(81 a, b)$ and $(82 a, b)$ by using $(6 a, b)$. According to (6a), the mean value of a quantitative character is the same as the mean genotypic value, whereas the variance of the character is obtained by adding the environmental variance, $v_{\mathrm{e}}$ to the genotypic value variance.

Notice, that no assumptions about the shape of the genotypic value distribution have been made in order to derive expressions $(81 \mathrm{a}, \mathrm{b})$ and $(82 a, b)$. The model is invariant with respect to the shape of the distribution.

A necessary condition for the trajectories described by $(81 \mathrm{a}, \mathrm{b})$ to converge to the equilibria $(82 \mathrm{a}, \mathrm{b})$ is that $\left|b_{1}\right|<\frac{1}{2}$ and $\left|a_{3}\right|<\frac{1}{2}$. These conditions are met if $b_{1}$ and $a_{3}$ are as given by (77a) and (77b):

$$
\begin{aligned}
& b_{1}=\frac{1}{2}\left(1-\mu_{+}-\mu_{-}\right), \\
& a_{3}=\left(\frac{1}{2}-\frac{n}{n-1} R\right)\left(1-\mu_{+}-\mu_{-}\right)^{2},
\end{aligned}
$$

with $0 \leqslant R<\frac{1}{2}$. Moreover, since $b_{1}$ and $a_{3}$ determined by these expressions are always positive, the trajectories $(81 \mathrm{a}, \mathrm{b})$ converge to the equilibria monotonically.

With $b_{1}$ and $b_{0}$ given in (77a), the equilibrium mean genotypic value is

$$
\hat{M}=2 n\left(\varepsilon_{1} \mu_{-}+\varepsilon_{0} \mu_{+}\right) /\left(\mu_{+}+\mu_{-}\right),
$$

and if the rates of forward and backward mutations are the same, i.e., $\mu_{+}=\mu_{-}$,

$$
\hat{M}=n\left(\varepsilon_{1}+\varepsilon_{0}\right) .
$$

The observed rates of mutations in different living organisms are usually very low. Consequently, for the mean genotypic value, the rate or approaching the equilibrium (85) determined by the term $2 b_{1}=1-\mu_{+}-\mu_{-}$ will usually be very slow. Therefore, if the dynamies of a quantitative character is considered during a relatively short period of time (say, only a few dozen generations), the role of mutations may be neglected, and it may be assumed that the mean genotypic value does not change during this time period, i.e.,

$$
M_{k}=M_{0}=M .
$$

The variance, on the other hand, may still change as

$$
V_{k}=\left(V_{1}-\hat{V}\right)\left(2 a_{3}\right)^{k}+\hat{V}
$$

approaching the equilibrium:

$$
\hat{V}=\left[\left(a_{3}+a_{2} / 1-\frac{1}{2}\right) M^{2}+2 a_{1} M+2 a_{0}\right] /\left(1-2 a_{3}\right) .
$$


When the role of mutations is neglected, the coefficients of the gametic model are given by (76a, b), and expressions (88), (89) take the form

$$
\begin{aligned}
& V_{k}=\left(V_{1}-\hat{V}\right)\left(1-\frac{2 n}{n-1} R\right)^{k}+\hat{V}, \\
& \hat{V}=-\frac{1}{2 n} M^{2}+\left(\varepsilon_{1}+\varepsilon_{0}\right) M-2 n \varepsilon_{1} \varepsilon_{0} .
\end{aligned}
$$

In terms of the traditional genic model, expression (90) would be regarded as describing the dynamics of the genotypic value variance in a population moving toward the state of linkage equilibrium, and the equilibrium value (91) would be regarded as the linkage equilibrium value of the variance.

Figures 2 and 3 illustrate the dynamics of the genotypic value variance during the first 50 generations of random mating for different numbers of loci and different recombination rates for a polygenic additive character with allelic contributions as 0 or 1 . The mutation rates in all cases where chosen to be $\mu_{+}=\mu_{-}=10^{-4}$ per locus, per generation. The ordinates of both figures represent the ratio of the variance of genotypic values in a given generation to its equilibrium value, which in all cases is equal to $n / 2$. Graphs for the dynamics of the phenotypic variances are easily obtained from Figures 2 and 3 by multiplying the ordinates by $n / 2$ and adding the corresponding values of the environmental variance, $v_{\mathrm{e}}$.

Figure 2 represents the case when the initial populations consist of only those types of gametes that have the same gametic effect, namely $n / 4$, i.e., it is assumed that $m_{0}=n / 4, v_{0}=0$ (or $M_{0}=n / 2, V_{0}=0$ ). It should be remem-

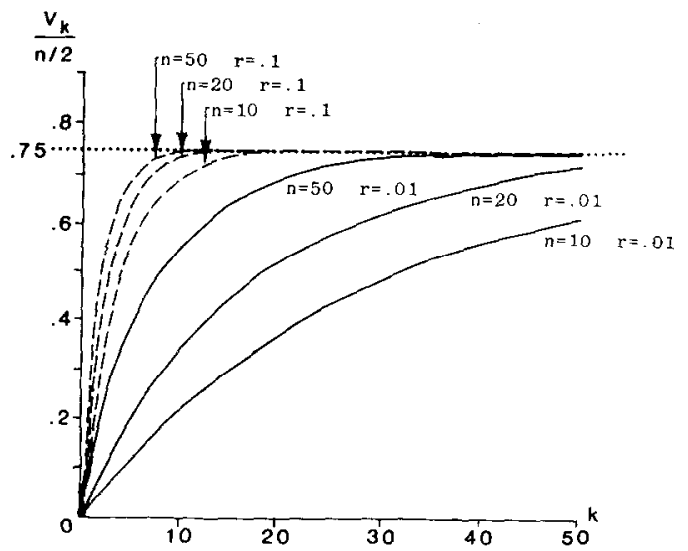

FIG. 2. Genotypic variance dynamics under random mating. I. Zero variance in the initial population ( $n$, number of loci; $r$, rate of recombination; $k$, generation number). 
bered that the zero variance of gametic effects does not imply that there is no variation of gametotypes. In the gametic model it only implies that no other gametotypes were present in the initial population, except for those whose gametic effect is $n / 4$, and that those present were in equal proportions.

The mean genotypic value in all cases did not change substantially during the 50 generations (no more than $1 \%$ increase as compared to the initial value). The variances (see Fig. 2) approached the linkage equilibrium value of 0.75 quite rapidly, but at different rates depending on the number of loci and rate of recombination. After 1000 generations of continued random mating, the variance approached $83 \%$ of the equilibrium value determined by mutations, and the mean changed to $70 \%$ of the equilibrium value. Notice, that for a fixed number of loci, an increase in the rate of recombination results in an accelerated change of the variance. With a fixed recombination rate, an increase in the number of loci also has an accelerating effect on the change of the variance.

Figure 3 represents the case when the initial populations were assumed to be such that one half of a population consisted of the individuals with the minimal genotypic value 0 and the other half of the individuals with the maximal genotypic value $2 n$. Thus, the initial values of the mean genotypic

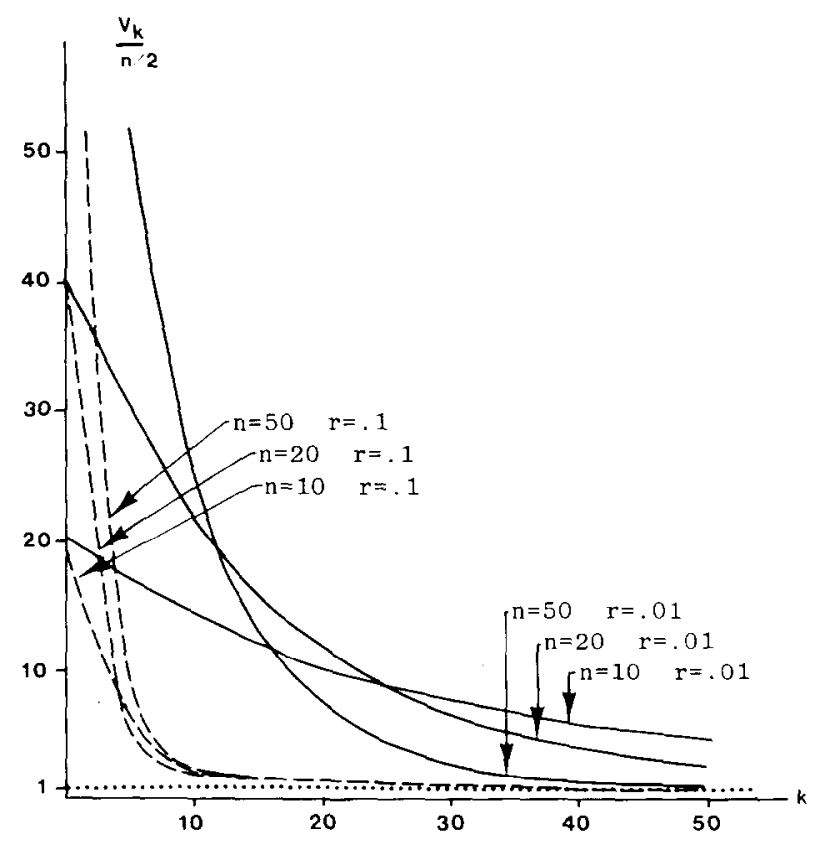

FIG. 3. Genotypic value variance dynamics under random mating. II. Maximal variance in the initial population ( $n$, number of loci; $r$, rate of recombination; $k$, generation number). 


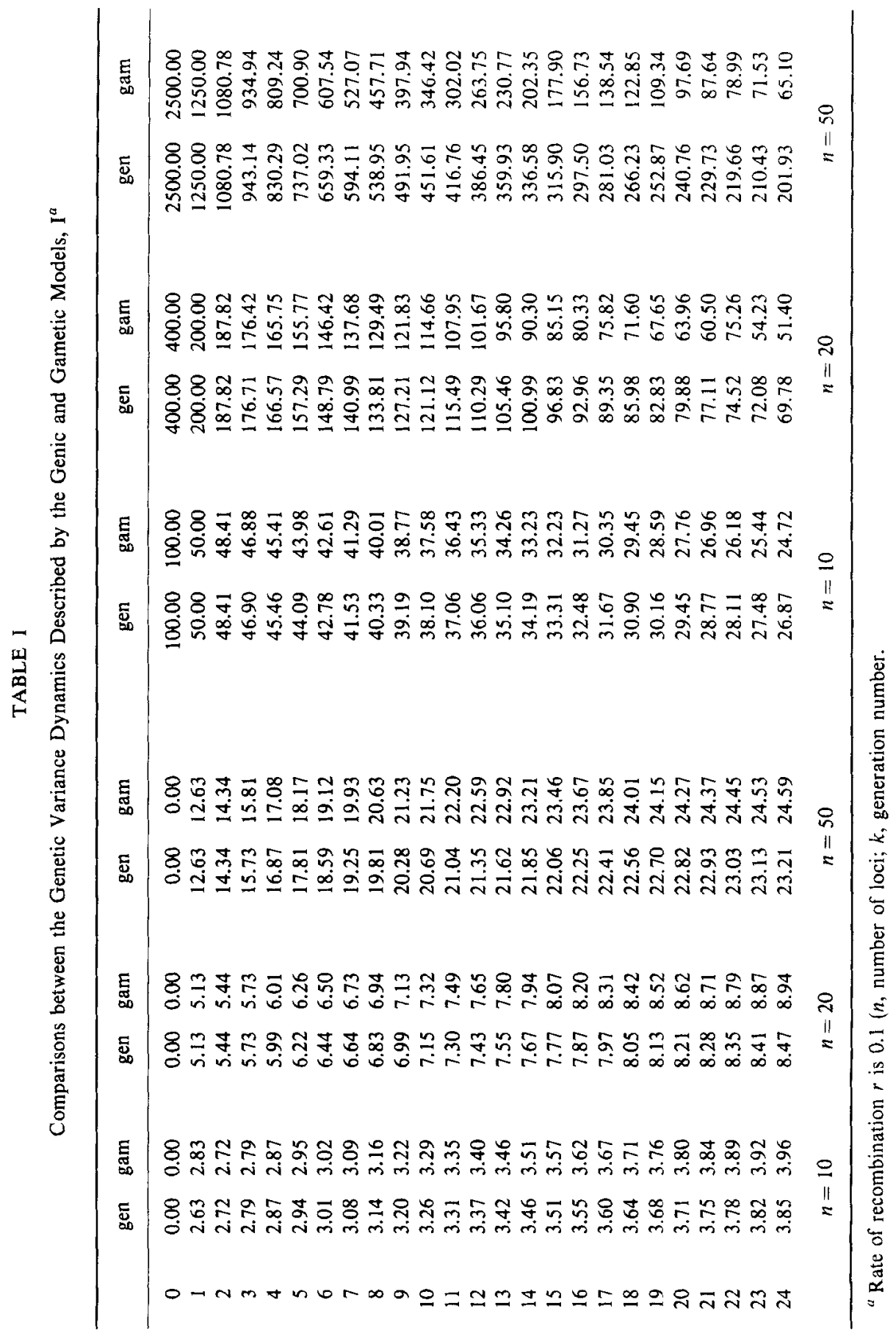


QUANTITATIVE CHARACTER DYNAMICS: GAMETIC MODEL

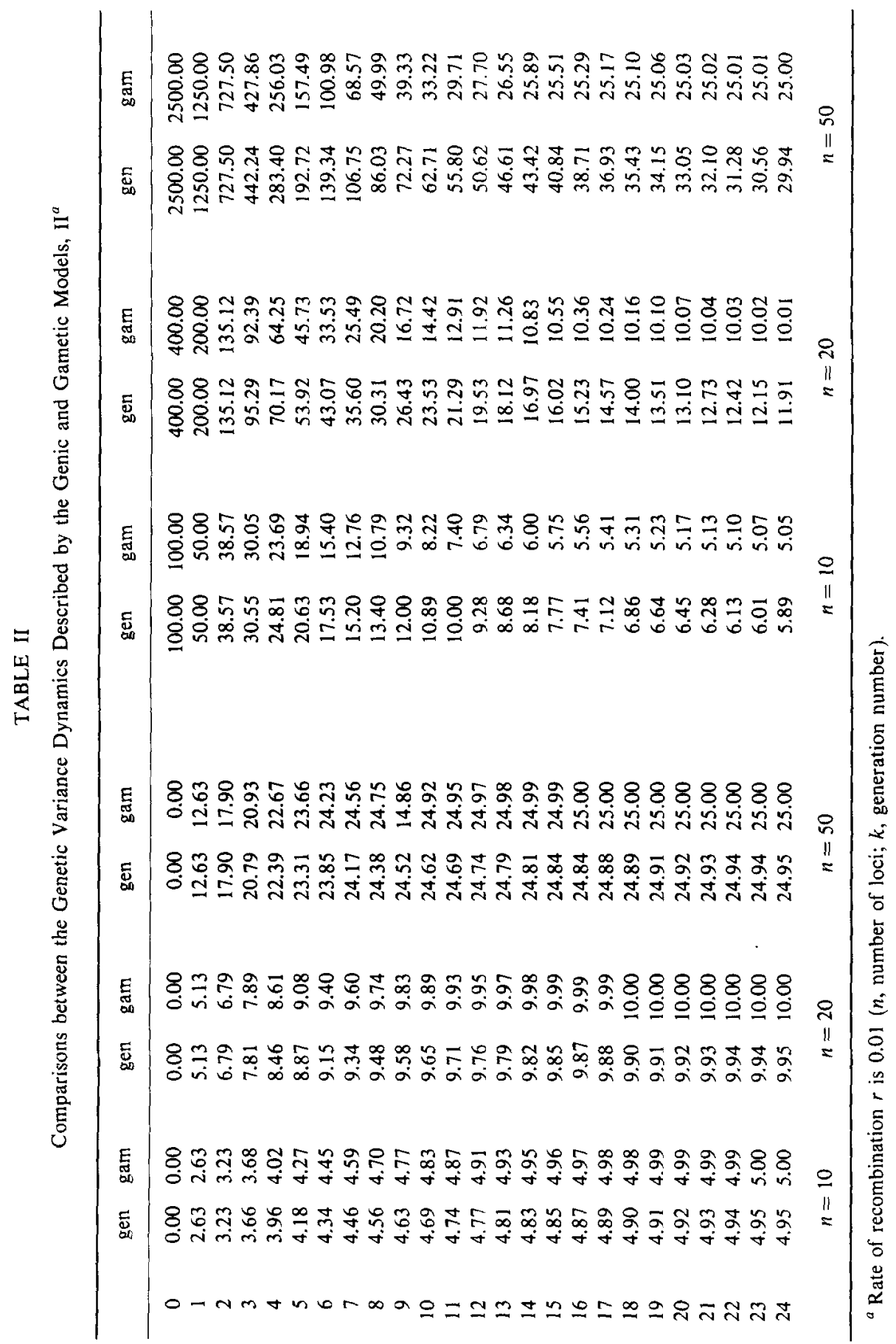


value were $M_{0}=n$ and of the variance, $V_{0}=n^{2}$. Since in this case the mean started at the equilibrium value, there was no change. The variance rapidly dropped to the equilibrium value of 1.0 on the ordinate scale. As in the previous case, an increase in either the number of loci or the recombination rate has an accelerating effect on the rate of change of the variance.

It is interesting to compare the dynamics of a polygenic additive character under random mating as predicted by the traditional genic model with that predicted by the gametic one. Tables I and II represent the dynamics of the genetic variance during 25 generations of random mating for a polygenic additive character controlled by $n$ loci when the rate of recombination between adjacent loci is $r$. Columns headed by "gen" represent the variance dynamics as described by the genic model (formulas 4.7.5-4.7.7 and 4.7.15 in Crow and Kimura, 1970). Columns "gam" represent the dynamics described by the gametic model shown in Figs. 2 and 3. The left halves of the tables correspond to the initial populations shown in Fig. 2, i.e., with zero initial variances, and the right halves of the tables correspond to the initial populations shown in Fig. 3, i.e., with the maximal initial variances. In Table $\mathrm{I}$, the rate of recombination, $r$ is 0.1 , and in Table II, it is 0.01 . There is a good correspondence between the dynamics described by the two types of models. The only exception is the case in Table II of the dynamics of a character controlled by 50 loci with a low rate of recombination $(r=0.01)$, when the initial population has the maximum variance. In general, the dynamics described by the gametic model are slightly faster than the dynamics described by the genic model.

Let us now turn to the dynamics under random mating of sex-dependent characters. As it has been mentioned earlier, we shall assume that the mutational processes in males are the same as in females. Therefore, the coefficients $b_{i}$ for different sexes are as in (78) for sex-controlled characters, and as in (79) for sex-linked.

The dynamical equations for the mean and variance of the genotypic values among malcs for a sex-controlled character follow from (23) and (24):

$$
\begin{aligned}
M_{k+1}^{*} & =2 b_{1} M_{k}^{*}+2 b_{0}, \\
V_{k+1}^{*} & =2 \bar{a}_{3} V_{k}^{*}+\left(\bar{a}_{3}+\bar{a}_{2} / 2-2 b_{1}^{2}\right)\left(M_{k}^{*}\right)^{2}+2\left(\bar{a}_{1}-2 b_{1} b_{0}\right) M_{k}^{*}+2\left(\bar{a}_{0}-b_{0}^{2}\right),
\end{aligned}
$$

where $\bar{a}_{i}$ are the "sex-averaged" coefficients

$$
\bar{a}_{i}=\frac{1}{2}\left(a_{i}^{*}+a_{i}^{* *}\right) \quad(i=0,1,2,3) .
$$

The dynamics of the mean and variance of the genotypic values among females can be obtained in any generation by using (25) and (26). 
Notice that Eqs. (92) and (93) are analogous to Eqs. (80a, b) for the dynamics of the genotypic value mean and variance in the case of sexindependent characters. The fact that the dynamics in both sexes are determined by "sex-averaged" parameters implies that observations on the dynamics of a sex-controlled character under random mating cannot reveal whether the gametogenetic processes in males and females are the same or not.

When considering sex-linked characters, let us follow the dynamics of the gametic effects rather than of the genotypic values. The system of dynamical equations for the mean gametic effects among the gametes received by individuals from their fathers and mothers follows from (43a) and (44a) as

$$
m_{k+1}^{*}=2 b_{1} m_{k}^{*}+b_{0}, \quad m_{k+1}^{* *}=b_{1}\left(m_{k}^{*}+m_{k}^{* *}\right)+b_{0} .
$$

The solution of this system is easily obtained:

$$
\begin{aligned}
m_{k}^{*} & =\left(\frac{1}{3} m_{0}^{*}+\frac{2}{3} m_{0}^{* *}\right)\left(2 b_{1}\right)^{k}+\frac{2}{3}\left(m_{0}^{*}-m_{0}^{* *}\right)\left(-b_{1}\right)^{k}+\hat{m}, \\
m_{k}^{* *} & =\left(\frac{1}{3} m_{0}^{*}+\frac{2}{3} m_{0}^{* *}\right)\left(2 b_{1}\right)^{k}-\frac{1}{3}\left(m_{0}^{*}-m_{0}^{* *}\right)\left(-b_{1}\right)^{k}+\hat{m},
\end{aligned}
$$

where

$$
\hat{m}=b_{0} /\left(1-2 b_{1}\right)
$$

For $b_{1}<\frac{1}{2}$, the mean gametic effects of the gametes received by individuals from their fathers and mothers converge, in the case of random mating, to the same equilibrium value (97). If $m_{0}^{*} \neq m_{0}^{* *}$, the convergence is not monotonic, and damped oscillations occur. Mean genotypic values among males and females can be computed by using formulas (45a) and (46a). It is obvious that both means converge to equilibrium values which are

$$
\begin{aligned}
\hat{M}^{*} & =b_{0} /\left(1-2 b_{1}\right) & & \text { (for males), } \\
\hat{M}^{* *} & =2 C b_{0} /\left(1-2 b_{1}\right) & & \text { (for females), }
\end{aligned}
$$

and the convergence can be accompanied by damped uscillations. If mutations are neglected, then $b_{1}=\frac{1}{2}, b_{0}=0$, and the dynamics of the mean gametic effects are described by the system of equations following from (95):

$$
m_{k+1}^{*}=m_{k}^{* *}, \quad m_{k+1}^{* *}=\frac{1}{2}\left(m_{k}^{*}+m_{k}^{* *}\right) .
$$

The solution of this system is

$$
\begin{aligned}
m_{k}^{*} & =\left(\frac{1}{3} m_{0}^{*}+\frac{2}{3} m_{0}^{* *}\right)+\frac{2}{3}\left(m_{0}^{*}-m_{0}^{* *}\right)\left(-\frac{1}{2}\right)^{k}, \\
m_{k}^{* *} & =\left(\frac{1}{3} m_{0}^{*}+\frac{2}{3} m_{0}^{* *}\right)-\frac{1}{3}\left(m_{0}^{*}-m_{0}^{* *}\right)\left(-\frac{1}{2}\right)^{k} .
\end{aligned}
$$


System (99) is analogous to the system of equations for the dynamics of the frequencies of sex-linked genes under random mating (see Crow and Kimura, 1970).

When analyzing the dynamics of the variance of a sex-linked character, let us neglect the mutation process, assuming that the coefficients $b_{i}$ and $a_{i}^{*}$ take the values

$$
b_{1}=\frac{1}{2}, \quad b_{0}=0 ; \quad a_{3}^{*}=1, \quad a_{1}^{*}=a_{0}^{*}=0 ;
$$

with the rest of the coefficients remaining as free parameters. Let us also assume for simplicity that the mean gametic effects are at the equilibrium:

$$
m_{k}^{*}=m_{k}^{* *}=\hat{m}
$$

Then, the system of cquations for the variances of the gametic effects follows from (43b) and (44b) as

$$
\begin{aligned}
& v_{k+1}^{*}=v_{k}^{* *}, \\
& v_{k+1}^{* *}=a_{3}^{* *}\left(v_{k}^{*}+v_{k}^{* *}\right)+\left(2 a_{3}^{* *}+a_{2}^{* *}-1\right) \hat{m}^{2}+2 a_{1}^{* *} \hat{m}+a_{0}^{* *} .
\end{aligned}
$$

The general solution of this system can be written in the form

$$
v_{k}^{*}=C_{1}\left(\lambda_{1}\right)^{k}+C_{2}\left(\lambda_{2}\right)^{k}+\hat{v}, \quad v_{k}^{* *}=C_{3}\left(\lambda_{1}\right)^{k}+C_{4}\left(\lambda_{2}\right)^{k}+\hat{v},
$$

where

$$
\hat{v}=\left[\left(2 a_{3}^{* *}+a_{2}^{* *}-1\right) \hat{m}^{2}+2 a_{1}^{* *} \hat{m}+a_{0}^{* *}\right] /\left(1-2 a_{3}^{* *}\right),
$$

$C_{i}$ are constants determined by the initial values of the variances, and $\lambda_{1}, \lambda_{2}$ are the characteristic roots

$$
\lambda_{1}=\left[a_{3}^{* *}+\sqrt{\left(a_{3}^{* *}\right)^{2}+4 a_{3}^{* *}}\right] / 2, \quad \lambda_{2}=\left[a_{3}^{* *}-\sqrt{\left(a_{3}^{* *}\right)^{2}+4 a_{3}^{* *}}\right] / 2 .
$$

It is easy to see that for $a_{3}^{* *}$ as in (76b),

$$
0 \leqslant \lambda_{1} \leqslant 1, \quad-\frac{1}{2} \leqslant \lambda_{2} \leqslant 0 .
$$

It follows then from (102) that the variances of the gametic effects among gemetes received by individuals from their fathers and mothers converge under random mating to the same equilibrium value (103). Since one of the characteristic roots, $\lambda_{2}$ is negative, this convergence can be accompanied by damped oscillations. Notice that these oscillations of the variance may take place even if the mean value of the gametic effects is at equilibrium. In practice, however, their amplitude will usually be very small, and they can hardly be noticed in real populations. 


\section{Discussion}

When evaluating the significance of different "macro" models of the quantitative character dynamics, one should not think of them as just generalizations of the traditional genic "micro" models, but rather as alternative approximations of dynamics of real biological populations using different levels of specification of the biological mechanisms. It should be kept in mind that no genic model of a quantitative character has ever been unambiguously confirmed experimentally. Therefore, models of this type, in spite of their long history, cannot be regarded as sacrosanct and serve as the ultimate basis for evaluating models of other types. It is important not to forget that genic models represent only one possible approach to approximating biological reality and are not the reality itself.

In the traditional genic models, the gene is the elementary unit of evolution, and gametes are characterized by their gametotypes (genotypes). What precisely is meant by the gametotype depends in each case on what is believed to be the concrete genetic system underlying a particular quantitative character: ploidy, number of loci, number of alleles, etc. If, for example, a character is believed to be under control of $n$ diploid, $m$-allelic loci, then an $n$-dimensional vector is used to represent a gametotype, and the total number of distinctive gametotypes is $m^{n}$. The hereditary mechanisms are then usually described in terms of replacements and substitutions of vector components (alleles). The genotypic value of an individual is considered to be a function defined on the set of pairs of gametotypes. Because of the complexity of hereditary and developmental processes in living organisms and formidable difficulties in experimental work, the genetic system of a real quantitative character almost always remains unknown. Consequently, the function determining the genotypic values of individuals is almost never specified. Moreover, even if this function could be specified, it certainly would be very complex, varying greatly for different characters in different organisms. Since the function itsclf takcs numerical values, whereas its arguments are vectors, it is quite difficult to find an approximation for it which, while being universal for different quantitative characters, would at the same time be mathematically convenient. (The traditional concepts of additive, dominant, and epistatic gene actions represent an attempt at such a universal in genic models approximation, which unfortunately is inconvenient mathematically.) It is even more difficult to find a mathematically convenient approximation to describe the hereditary mechanisms in genic models.

In the gametic model presented in this paper, gametes are treated as the elementary units and are characterized by gametic effects rather than by their gametotypes. Just as the genotypic value of an individual, usually defined as the "contribution to the quantitative character due to the 
individual's genotype"is a mathematical abstraction with no direct biological meaning, the gametic effect is also a mathematical abstraction. It may be regarded simply as a number assigned to a gametotype according to some hypothetical rule. The genotypic value of an individual is then a function (developmental function $f(\alpha, \beta)$ ) defined on the set of pairs of gametic effects, whereas hereditary mechanisms are described by the gametogenetic function $H(\lambda \mid \alpha, \beta)$, whose domain consists of trios of gametic effects. The particular form and properties of the developmental and gametogenetic functions will depend on the rule assigning gametic effects to different gametotypes. If the rule is assumed to be such that a one-to-one relation between gametotypes and gametic effects is established (e.g., simple enumeration of all possible gametotypes), the corresponding developmental and gametogenetic functions, although almost certainly very complex, will at the same time be independent of the genetic structure of the population and remain unchanged in the process of the population dynamics. Expression (3) would in such a case provide an exact description of the dynamics of the gametic effect distribution. The model, however, will almost surely be too complicated for an analytical or numerical treatment, and some approximations of the developmental and gametogenetic functions (or of the first, $m_{H}(\alpha, \beta)$, and the second, $m_{H}^{\prime \prime}(\alpha, \beta)$, moments of the gametogenetic function, if only the dynamics of the mean and variance of the character are of the main interest) need to be found. Since $f(\alpha, \beta), m_{H}(\alpha, \beta)$, and $m_{H}^{\prime \prime}(\alpha, \beta)$ are numerical functions of numerical arguments, they can be subjected to approximations by mathematical expressions, and by linear and quadratic polynomials in particular. How good the polynomials are as approximations will, of course, depend on the particularities of the genetic system of the quantitative character and on the hypothetical rule assigning gametic effects to gametotypes. Notice, however, that the gametic effects enter the model not as explicit parameters, but rather as its "internal" variables, and neither gametotypes nor the rule assigning gametic effects are specified. Therefore, it may always be assumed that for any quantitative character, the gametotypes and the rule, although remaining unknown to us, are such that the corresponding functions $f(\alpha, \beta), m_{H}^{\prime \prime}(\alpha, \beta)$, and $m_{H}^{\prime \prime}(\alpha, \beta)$ are "the best" for being approximated by the polynomials, as compared to all other gametotypes and rules imaginable for the genetic system of the character. The predictive value of a gametic model based on the representation of these functions by linear and quadratic polynomials will, of course, vary for different characters in different populations. Even for the same character in the same population the accuracy of the prediction may change in the process of population dynamics and become inadequate in the long run.

While keeping in mind that the gametic model should not be viewed as just a generalization of the genic one, it is of interest to know whether the two types of models are related. It is indeed always desirable that a 
continuity and some degree of overlap exist between models dealing with similar problems at different levels, especially if there is a lack of experimental verification for either of them. Moreover, an overlap between different types of models may help in making inferences about the parameters of a higher level model, based on a lower level model, when these parameters cannot be or have not been identified experimentally.

The concept of polygenic additive characters establishes a continuity between the traditional genic and the gametic models. The rule assigning gametic effects to gametotypes in this case is such that the gametic effect is the sum of the components of the vector representing a gametotype. The corresponding developmental function is then simply the sum of the gametic effects and, obviously, does not change from generation to generation. As for the corresponding gametogenetic function accounting for mutations and crossing-over, it cannot be expected to remain unchanged. If the population of genotypes in the genic model is regarded deterministically, the gametogenetic function depends on the actual distribution of gametotypes in the population. Thus, if the population is regarded deterministically, dynamics of a quantitative character, as described by a gametic model, will in general differ from the dynamics described by a genic mode. It was demonstrated, however, that when free recombination or complete linkage between all loci is assumed, the gametic and genic models are equivalent, even when treating a population as deterministic. On the other hand, when treating a population statistically and using the genic model, the gametogenetic function corresponding to a polygenic additive character becomes independent of the population genetic structure and, hence, remains unchanged from generation to generation. In this case, the first and second moments of the gametogenetic function become exactly first- and seconddegree polynomials, and the gametic and genic models are totally equivalent. By treating the population of genotypes in a genic model statistically (which is tantamount to assuming that all gametotypes with the same gametic effect are present in the population in equal frequencies), we implicitly admit that, besides the processes accounted for by the model, there also may exist some other processes changing the population genetic structure randomly. Although only experimental data can tell which of the concepts best approximates biological reality, it is quite probable that in many cases a population of genotypes treated statistically is closer to approximating the reality than the same population treated deterministically. A somewhat similar statistical concept of a population of genotypes has been employed by Ginzburg and Braumann (1980) in a problem concerning the relative importance of selection and recombination in multilocus genetic systems. 


\section{APPENDIX}

First, let us prove that

$$
\sum_{i=1}^{n} \operatorname{Pr}\left[\alpha_{i}=1 \mid \alpha\right]=\alpha
$$

This can be proven by the method of induction. Notice, that (A1) holds for $n=1$. Assume now that it holds for an arbitrary $n$, and let us show that it then will also hold for $n+1$. Indeed,

$$
\begin{aligned}
\sum_{i=1}^{n+1} \operatorname{Pr}\left[\alpha_{i}=1 \mid \alpha\right]= & \operatorname{Pr}\left[\alpha_{n+1}=0 \mid \alpha\right] \sum_{i=1}^{n} \operatorname{Pr}\left[\alpha_{i}=1 \mid \alpha\right] \\
& +\operatorname{Pr}\left[\alpha_{n+1}=1 \mid \alpha\right] \sum_{i=1}^{n} \operatorname{Pr}\left[\alpha_{i}=1 \mid \alpha-1\right] \\
& +\operatorname{Pr}\left[\alpha_{n+1}=1|\alpha| .\right.
\end{aligned}
$$

According to the assumption that (A1) holds for a fixed value of $n,(\mathrm{~A} 2)$ can be transformed as

$$
\begin{aligned}
\sum_{i=1}^{n+1} \operatorname{Pr}\left[\alpha_{i}=1 \mid \alpha\right]= & \alpha \operatorname{Pr}\left[\alpha_{n+1}=0|\alpha|+(\alpha-1) \operatorname{Pr}\left[\alpha_{n+1}=1|\alpha|\right.\right. \\
& +\operatorname{Pr}\left|\alpha_{n+1}=1\right| \alpha \mid .
\end{aligned}
$$

Since $\operatorname{Pr}\left[\alpha_{n+1}=0 \mid \alpha\right]+\operatorname{Pr}\left[\alpha_{n+1}=1 \mid \alpha\right]=1$, (A3) is reduced to

$$
\sum_{i=1}^{n+1} \operatorname{Pr}\left[\alpha_{i}=1 \mid \alpha\right]=\alpha,
$$

thus concluding the proof that (A1) holds for any $n$.

Second, let us prove that

$$
\sum_{i \neq j}^{n} \operatorname{Pr}\left[\alpha_{i}=1, \alpha_{j}=1 \mid \alpha\right]=\alpha(\alpha-1) .
$$

It is easy to see that this holds for $n=2$ by noticing that the left-hand side of (A5) is reduced in this case to

$$
\begin{aligned}
\operatorname{Pr}\left[\alpha_{1}\right. & \left.=1, \alpha_{2}=1 \mid \alpha_{1}+\alpha_{2}=\alpha\right]+\operatorname{Pr}\left[\alpha_{2}=1, \alpha_{1}=1 \mid \alpha_{1}+\alpha_{2}=\alpha\right] \\
& =2 \operatorname{Pr}\left[\alpha_{1}=1, \alpha_{2}=1 \mid \alpha_{1}=\alpha_{2}=\alpha\right],
\end{aligned}
$$


which is equal to 0 when $\alpha=0$ or $\alpha=1$, and is equal to 2 when $\alpha=2$. Assume now that (A5) holds for an arubtrary $n \geqslant 2$, and let us show that it then will hold for $n+1$. Indeed,

$$
\begin{aligned}
\sum_{i \neq j}^{n+1} \operatorname{Pr}\left[\alpha_{i}=1, \alpha_{j}=1 \mid \alpha\right]= & \operatorname{Pr}\left[\alpha_{n+1}=0 \mid \alpha\right] \sum_{i \neq j}^{n} \operatorname{Pr}\left[\alpha_{i}=1, \alpha_{j}=1 \mid \alpha\right] \\
& +\operatorname{Pr}\left[\alpha_{n+1}=1 \mid \alpha\right]\left(\sum _ { i \neq j } ^ { n } \operatorname { P r } \left[\alpha_{i}=1, \alpha_{j}=1|\alpha-1|\right.\right. \\
& +\sum_{i=1}^{n} \operatorname{Pr}\left[\alpha_{i}=1 \mid \alpha-1\right] \\
& +\sum_{j=1}^{n} \operatorname{Pr}\left[\alpha_{j}=1|\alpha-1|\right),
\end{aligned}
$$

or, using the assumption that (A5) holds for a fixed value of $n$,

$$
\begin{aligned}
\sum_{i \neq j}^{n+1} \operatorname{Pr}\left[\alpha_{i}=1, \alpha_{j}=1 \mid \alpha\right]= & \alpha(\alpha-1) \operatorname{Pr}\left[\alpha_{n+1}=0 \mid \alpha\right]+((\alpha-1)(\alpha-2) \\
& +2(\alpha-1)) \operatorname{Pr}\left[\alpha_{n+1}=1 \mid \alpha\right] \\
= & \alpha(\alpha-1)\left(\operatorname{Pr}\left[\alpha_{n+1}=0 \mid \alpha\right]+\operatorname{Pr}\left[\alpha_{n+1}=1 \mid \alpha\right]\right) \\
= & \alpha(\alpha-1),
\end{aligned}
$$

thus completing the proof that (A5) holds for any $n \geqslant 2$.

Third, let us consider properties of the parameter $R$ as a function of the number of loci and recombination rate. Let us recall that this parameter is (see (73))

$$
R=z_{n} / 2 n^{2},
$$

where $n$ is the number of loci and

$$
z_{n}=\sum_{i \neq j}^{n} r_{i j} \quad(n>1),
$$

with $r_{i j}$ being the probability of recombination between loci $i$ and $j$. Let us now introduce a new variable,

$$
y_{n}=\sum_{i=1}^{n} r_{i n} .
$$

It is not difficult to see that this variable follows the recursive relation:

$$
y_{n+1}=y_{n}\left(1-2 r_{n}\right)+n r_{n},
$$


where $r_{n}$ is the coefficient of recombination between adjacent loci $n$ and $n+1$. This is the same formula, of course, that is used in the derivation of the gene map distances (see Bailey, 1961). It is also not difficult to see that, given the value of variable $z$ for a fixed $n$, the value of $z$ for $n+1$ can be represented as

$$
z_{n+1}=z_{n}+2 y_{n+1}
$$

Thus, in order to obtain $z$ as a function of the number of loci and recombination coefficients, the following system of recursive equations ought to be solved:

$$
z_{n+1}=z_{n}+2 y_{n+1}, \quad y_{n+1}=y_{n}\left(1-2 r_{n}\right)+n r_{n}
$$

under the initial condition: $z_{1}=0, y_{1}=0$. A solution is easily obtained in the case when the recombination coefficients between all adjacent loci are the same (i.e., $r_{n}=r$ for any $n$ ). This solution yields the following formula for the parameter $R$ :

$$
R=\left[1-2 r(1-r n)(n+1)-(1-2 r)^{n+1}\right] / 8 r^{2} n^{2} .
$$

Figure 1 illustrates how $R$ depends on the number of loci for different recombination coefficients. It may take values between 0.0 and 0.25 . When recombination is low ( $r$ is of the order of 0.5 to $1 \%$ ), the value of $R$ is close to zero even for relatively large number of loci. When recombination is high $(r$ is of the order of 25 to $50 \%), R$ approaches its limiting value of 0.25 even for a relatively small number of loci.

\section{ACKNOWLEDGMENTS}

This work was initiated while I was with the Agrophysical Institute in Leningrad, and I would like to use this opportunity to thank those of my colleagues there who provided me with their help and encouragement. A part of this work has appeared in my doctoral thesis at the University of Wisconsin in Madison, and I acknowledge K. Aoki, J. Bull, J. Crow, W. Engels, and R. I,ande for their assistance and critical discussions. I also thank the referees for their valuable criticism.

\section{REFERENCES}

Balley, N. T. J. 1961, "An Introduction to the Mathematical Theory of Genetic Linkage," Oxford Univ. Press, London/New York.

Bulmer, M. G. 1971. The effect of selection on genetic variability, Amer. Natur. 105, 201-211.

BULmer, M. G. 1972. The genetic variability of polygenic characters under optimizing selection, mutation and drift, Genet. Res. 19, 17-25. 
BULMER, M. G. 1973. The maintenance of the genetic variability of polygenic characters by heterozygous advantage, Genet. Res. 22, 9-12.

Cavalli-Sforza, L. L., and Feldman, M. W. 1976. Evolution of continuous variation: direct approach through joint distribution of genotypes and phenotypes, Proc. Natl. Acad. Sci. USA 73, 1689-1692.

Caval.li-Sfor7a, L. L., and Feldman, M. W. 1978. The evolution of continuous variation. III. Joint transmission of genotype, phenotype and environment, Genetics 90, 391-425.

Cloninger, C. R., Rice, J., AND Reich, T. 1979. Multifactorial inheritance with cultural transmission and assortative mating. II. A general model of combined polygenic and cultural inheritance, Amer. J. Hum. Genet. 31, 176-198.

Crow, J. F., ANd Felsenstein J. 1968. The effect of assortative mating on the genetic composition of a population, Eugen. Quart. 15, 85-97.

Crow, J. F., AND Kimura, M. 1970. "An Introduction to Population Genetics Theory," Harper \& Row, New York/Evanston/London.

Feldman, M. W., and Cavalli-Sforza, L. L. 1977. The evolution of continuous variation. II. Complex transmission and assortative mating, Theor. Pop. Biol. 11, 161-181.

Feldman, M. W., and Cavalli-Sforza, L. L. 1979. Aspects of variance and covariance analysis with cultural inheritance, Theor. Pop. Biol. 15, 276-307.

Feldman, M. W., and Cavalli-Sforza, L. L. 1981. Assortative mating, selection and mutation models for continuous variation: A reply to Felsenstein, Theor. Pop. Biol. 19, $391-425$.

FELSENSTEIN, J. 1977. Multivariate normal genetic models with a finite number of loci, in Proceedings of the International Conference on Quantitative Genetics, August 16-21, 1976" (E. Pollak, O. Kempthorne, and T. B. Bailey, Jr., Eds.), pp. 227-246, lowa State Univ. lowa City.

Felsenstein, J. 1981. Continuous-genotype and assortative mating, Theor. Pop. Biol. 19, $341-357$.

Fleming, W. H. 1979. Equilibrium distribution of continuous polygenic traits, SIAM J. Appl. Math. 36, 148-168.

GimelfArB, A. 1970. The limiting distribution of a character in populations with the normal inheritance function, in Bull. Sci. Infor. Agronom. Phys., No. 14, Leningrad (in Russian).

Ginzburg, L., AND BraumanN, C. A. 1980. Multilocus population genetics: Relative importance of selection and recombination, Theor. Pop. Biol. 17, 298-320.

KARLIN, S. 1977. Selection with many loci and possible relations to quantitative genetics, in Proceedings of the International Conference on Quantitative Genetics, August 16-21, 1976 (E. Pollak, O. Kempthorne, and T. B. Bailey, Jr. Eds.), pp. 207-226, Iowa State Univ.

KAKLIN, S. 1978. Theoretical aspects of multilocus selection balance, in Studies in Mathematical Biology (S. Levin, Ed.), pp. 503-587, Math. Assoc. Amer.

KARLIN, S. 1979a. Models for multifactorial inheritance. I. Multivariate formulations and basic convergence result, Theor. Pop. Biol. 15, 308-355.

KARLiN, S. 1979b. Models for multifactorial inheritance. II. The covariance structure for a scalar phenotype under selective assortative mating and sex-dependent symmetric parentaltransmission, Theor. Pop. Biol. 15, 356-393.

KARLIN, S. 1979c. Models for multifactorial inheritance. III. Calculation of covariance of relatives under extended selective mating mechanisms, Theor. Pop. Biol. 15, 394-423.

KARLIN, S. 1979d. Models for multifactorial inheritance. IV. Asymmetric transmission for a scalar phenotype, Theor. Pop. Biol. 15, 424-438.

KARLiN, S. 1980a. Models of multifactorial inheritance. V. Linear assortative mating as against selective (nonlinear) assortative mating, Theor. Pop. Biol. 17, 255-275.

KARLIN, S. 1980b. Models of multifactorial inheritance. VI. Formulas and properties of the vector phenotype equilibrium covariance matrix, Theor. Pop. Biol. 17, 276-297. 
KARLIN, S. AND LIBERMAN, U. 1979. Central equilibria in multilocus systems. I. Generalized nonepistatic selection regimes, Genetics 91, 777-798.

KimURA, M. 1965. A stochastic model concerning the maintenance of genetic variability in quantitative characters. Proc. Natl. Acad. Sci. USA 54, 731-736.

LANDE, R. 1976a. The maintenance of genetic variability by mutations in polygenic character with linked loci. Genet. Res. 26, 221-235.

LANDE, R. 1976b. Natural selection and random genetic drift in phenotypic evolution, Evolution 30, 314-334.

LATTER, B. D. H. 1970. Selection in finite populations with multiple alleles. II. Centripetal selection, mutation and isoallelic variation, Genetics 66, 165-186.

LATTER, B. D. H. 1972. Selection in finite populations with multiple alleles. III. Genetic divergence with centripetal selection and mutation, Genetics 70, 475-490.

LEwontin, R. C. 1964a. The interaction of selection and linkage. I. General considerations: Heterotic models, Genetics 49, 49-67.

LEwONTIN R. C. 1964b. The interaction of selection and linkage. II. Optimum models. Genetics 50, 757-782.

Lewontin, R. C. 1974. "The Genetic Basis of Evolutionary Change,"Columbia Univ. Press, New York/London.

Rice, J., Cloninger, C. R., AND Reich, T. 1978. Multifactorial inheritance with cultural transmission and assortativc mating. 1. Description and basic properties of the unitary models, Amer. J. Human. Genet. 30, 618-643.

Rocklin, S., AND Oster, G. 1976. Competition between phenotypes, J. Math. Biol. 3, 225-261.

Slatkin, M. 1970. Selection and polygenic characters, Proc. Natl. Acad. Sci. USA 66, 78-93.

SLATKIN, M. 1979. Frequency and density dependent selection on a quantitative character, Genetics 93, 755-771.

SLATKIN, M., AND LANDE, R. 1976. Niche width in a fluctuating environment-density independent model, Amer. Natur. 119, 341-357.

Wright, S. 1921a. Systems of mating. II. The effects of inbreeding on the genetic composition of a population. Genetics 6, 124-143.

WRIGHT, S. 1921 b. Systems of mating. III. Assortative mating based on somatic resemblance, Genetics 6, 144-161. 